\title{
THE ISING PARTITION FUNCTION: ZEROS AND DETERMINISTIC APPROXIMATION
}

\author{
JINGCHENG LIU, ALISTAIR SINCLAIR, AND PIYUSH SRIVASTAVA
}

\begin{abstract}
Aвs TRACT. We study the problem of approximating the partition function of the ferromagnetic Ising model with both pairwise as well as higher order interactions (equivalently, in graphs as well as hypergraphs). Our approach is based on the classical Lee-Yang theory of phase transitions, along with a new Lee-Yang theorem for the Ising model with higher order interactions, and on an extension of ideas developed recently by Barvinok, and Patel and Regts that can be seen as an algorithmic realization of the Lee-Yang theory.

Our first result is a deterministic polynomial time approximation scheme (an FPTAS) for the partition function in bounded degree graphs that is valid over the entire range of parameters $\beta$ (the interaction) and $\lambda$ (the external field), except for the case $|\lambda|=1$ (the "zero-field" case). A polynomial time randomized approximation scheme (FPRAS) for all graphs and all $\beta, \lambda$, based on Markov chain Monte Carlo simulation, has long been known. Unlike most other deterministic approximation algorithms for problems in statistical physics and counting, our algorithm does not rely on the "decay of correlations" property, but, as pointed out above, on Lee-Yang theory. This approach extends to the more general setting of the Ising model on hypergraphs of bounded degree and edge size, where no previous algorithms (even randomized) were known for a wide range of parameters. In order to achieve this latter extension, we establish a tight version of the Lee-Yang theorem for the Ising model on hypergraphs, improving a classical result of Suzuki and Fisher.
\end{abstract}

Jingcheng Liu, Computer Science Division, UC Berkeley. Email: 1iuexp@berkeley.edu. Alistair Sinclair, Computer Science Division, UC Berkeley. Email: sinclair@cs . berkeley.edu.

Piyush Srivastava, Tata Institute of Fundamental Research, Mumbai. Email: piyush.srivastava@tifr.res .in.

An extended abstract of this paper appeared in the proceedings of the 58th Annual IEEE Symposium on Foundations of Computer Science (FOCS), 2017. pp.986-997. 


\section{INTRODUCTION}

The Ising model, first studied a century ago as a model for magnetic materials by Lenz and Ising [25], has since become an important tool for the modeling of interacting systems. In the Ising model, such a system is represented as a graph $G=(V, E)$, so that the individual entities comprising the system correspond to the vertices $V$ and their pairwise interactions to the edges $E$. A configuration of the system is an assignment $\sigma: V \rightarrow\{+,-\}$ of one of two possible values (often called "spins") to each vertex. The model then induces a probability distribution (known as a Gibbs distribution) over these global configurations in terms of local parameters that model the interactions of the vertices.

In our setting, it will be convenient to parameterize these interactions in terms of a vertex activity or fugacity $\lambda$, that models an "external field" and determines the propensity of a vertex to be in the + configuration, and an edge activity $\beta \geq 0$ that models the tendency of vertices to agree with their neighbors. The model assigns to each configuration $\sigma$ a weight

$$
w(\boldsymbol{\sigma}):=\beta^{|\{\{u, v\} \in E \mid \boldsymbol{\sigma}(u) \neq \boldsymbol{\sigma}(v)\}|} \lambda^{|\{v \mid \boldsymbol{\sigma}(v)=+\}|}=\beta^{|E(S, \bar{S})|} \lambda^{|S|},
$$

where $S=S(\boldsymbol{\sigma})$ is the set of vertices assigned spin + in $\boldsymbol{\sigma}$ and $E(S, \bar{S})$ is the set of edges in the cut $(S, \bar{S})$ (i.e., the number of pairs of adjacent vertices assigned opposite spins). The probability of configuration $\boldsymbol{\sigma}$ under the Gibbs distribution is then $\mu(\boldsymbol{\sigma}):=w(\boldsymbol{\sigma}) / Z_{G}^{\beta}(\lambda)$, where the normalizing factor $Z_{G}^{\beta}(\lambda)$ is the partition function defined as

$$
Z_{G}^{\beta}(\lambda):=\sum_{\boldsymbol{\sigma}: V \rightarrow\{+,-\}} w(\boldsymbol{\sigma})=\sum_{S \subseteq V} \beta^{|E(S, \bar{S})|} \lambda^{|S|} .
$$

Notice that the partition function may be interpreted combinatorially as a cut generating polynomial in the graph $G$.

In this paper we focus on the original ferromagnetic case in which $\beta<1$, so that configurations in which a larger number of neighboring spins agree (small cuts) have higher probability. The anti-ferromagnetic regime $\beta>1$ is qualitatively very different, and prefers configurations with disagreements between neighbors. We note also that all our results in this paper hold in the more general setting where there is a different interaction $\beta_{e}$ on each edge, provided that all the $\beta_{e}$ satisfy whatever constraints we are putting on $\beta$. (So, e.g., in the ferromagnetic case $\beta_{e}<1$ for all $e$.) In addition, our results allow $\beta$ to be negative and $\lambda$ to be complex; this will be discussed in more detail below.

As in almost all statistical physics and graphical models, the partition function captures the computational complexity of the Ising model. Partition functions are $\# \mathrm{P}-$ hard $^{1}$ to compute exactly in virtually any interesting case (e.g., this is true for the Ising model except in the trivial cases $\lambda=0$ or $\beta \in\{0,1\}$ ), so attention is focused on approximation. An early result in the field due to Jerrum and Sinclair [27] establishes a fully polynomial randomized approximation scheme for the Ising partition function, valid for all graphs $G$ and all values of the parameters $(\beta, \lambda)$ in the ferromagnetic regime. Like many of the first results on approximating partition functions, this algorithm is based on random sampling and the Markov chain Monte Carlo method.

For several statistical physics models on bounded degree graphs (including the anti-ferromagnetic Ising model [30,47] and the "hard core", or independent set model [54]), fully-polynomial deterministic approximation schemes have since been developed, based on the decay of correlations property in those models: roughly speaking, one can estimate the local contribution to the partition function at a given vertex $v$ by exhaustive enumeration in a neighborhood around $v$, using decay of correlations to truncate the neighborhood at logarithmic diameter. The range of applicability of these algorithms is of course limited to the regime in which decay of correlations holds, and indeed complementary results prove that

\footnotetext{
${ }^{1}$ If a combinatorial counting problem, such as computing a partition function in a statistical physics model, is \#P-hard, then it can be solved in polynomial time only if all counting problems belonging to a very rich class can be solved in polynomial time. Hence \#P-hardness is widely regarded as compelling evidence of the intractibility of efficient exact computation. For a more detailed account of this phenomenon in the context of partition functions, see, e.g., [46, Appendix A].
} 
the partition function is NP-hard to approximate outside this regime [19,49]. Perhaps surprisingly, however, no deterministic approximation algorithm is known for the classical ferromagnetic Ising partition function that works over anything close to the full range of the randomized algorithm of [27]: to the best of our knowledge, the best deterministic algorithm, due to Zhang, Liang and Bai [56], is based on correlation decay and is applicable to graphs of maximum degree $\Delta$ only when $\beta>1-2 / \Delta$.

The restricted applicability of correlation decay based algorithms for the ferromagnetic Ising model arises from two related reasons: the first is that this model does not exhibit correlation decay for $\beta$ sufficiently close to 0 (for any given value of the external field), so any straightforward approach based only on this property cannot be expected to work for all $\beta$. Secondly, there is a regime of parameters for which, even though decay of correlation holds, there is evidence to believe that it cannot be exploited to give an algorithm using the usual techniques: see [47, Appendix 2] for a more detailed discussion of this point.

The first goal of this paper is to supply such a deterministic algorithm which covers almost the entire range of parameters of the model except for the "zero-field" case $|\lambda|=1$ :

Theorem 1.1. Fix any $\Delta>0$. There is a fully polynomial time approximation scheme (FPTAS) $)^{2}$ for the Ising partition function $Z_{G}^{\beta}(\lambda)$ in all graphs $G$ of maximum degree $\Delta$ for all edge activities $-1 \leq \beta \leq 1$ and all (possibly complex) vertex activities $\lambda$ with $|\lambda| \neq 1$.

Remarks. (i) For fixed $\Delta$ and $\lambda$ such that $|\lambda|<1$, the running time of the FPTAS for producing a $(1 \pm \varepsilon)$ factor approximation on $n$-vertex graphs of degree at most $\Delta$ is $(n / \varepsilon)^{O\left(\frac{\log \Delta}{|1-| \lambda \mid}\right)}$ (the running times of the algorithms in Theorems 1.3 and 1.4 have a similar dependence on $\lambda$ and $\Delta$ ). Such dependence on the "distance to the critical boundary" (in this case, the circle $|\lambda|=1$ ) of the degree of the polynomial bounding the running time of the FPTAS appears to be a common feature of algorithms based on correlation decay $[30,48,54]$ as well as our present analytic continuation approach. In contrast, approximate counting algorithms based on Markov chain Monte Carlo (e.g., $[18,26,34]$ ) often have the desirable feature that they are in a sense "fixed parameter tractable": even as the fixed parameters of the problems are moved close to the boundary up to which the algorithm is applicable, the degree of the polynomial bounding their running time does not increase; it is only the constant factors which increase to infinity. (ii) Note that although $\lambda, \beta$ are positive in the "physically relevant" range in most applications of the Ising model, the above theorem also applies more generally to $\beta \in[-1,1]$ and complex valued $\lambda$ not on the unit circle. Moreover, we can allow edge-dependent activities $\beta_{e}$ provided all of them lie in $[-1,1]$.

The above theorem is actually a special case of a more general theorem for the hypergraph version of the Ising model (Theorem 1.3 below). We now illustrate our approach to proving these theorems, which will also allow us to introduce and motivate our further results in the paper.

In contrast to previous algorithms based on correlation decay, our algorithm is based on isolating the complex zeros of the partition function $Z:=Z_{G}^{\beta}(\lambda)$ (viewed as a polynomial in $\lambda$ for a fixed value of $\beta$ ). This approach was introduced recently by Barvinok [6,7] (see also the recent monograph by Barvinok [8] for a discussion of the approach in a more general context). We start with the observation that the $1 \pm \varepsilon$ multiplicative approximation of $Z$ required for a FPTAS corresponds to a $O(\varepsilon)$ additive approximation of $\log Z$. Barvinok's approach considers a Taylor expansion of $\log Z$ around a point $\lambda_{0}$ such that $Z\left(\lambda_{0}\right)$ is easy to evaluate. (For the Ising model, $\lambda_{0}=0$ is such a choice.) It then seeks to evaluate the function at other points by carrying out an explicit analytic continuation. More concretely, suppose it can be shown that there are no zeros of $Z$ in the open disk $D\left(\lambda_{0}, r\right)$ of radius $r$ around $\lambda_{0}$. From standard results in complex analysis, it then follows that the Taylor expansion around $\lambda_{0}$ of $\log Z$ is absolutely convergent in $D\left(\lambda_{0}, r\right)$ and further, that the first $m$ terms of this expansion evaluated at a point $\lambda \in D\left(\lambda_{0}, r\right)$ provide

\footnotetext{
${ }^{2}$ A FPTAS takes as input an $n$-vertex (hyper)graph $G$, model parameters $\beta, \lambda$, and an accuracy parameter $\varepsilon \in(0,1)$ and outputs a value that approximates $Z_{G}^{\beta}(\lambda)$ within a factor $1 \pm \varepsilon$ (see also eq. (3)). The running time of the algorithm is polynomial in $n$ and $1 / \varepsilon$.
} 
a $O\left(\frac{n \alpha^{m}}{1-\alpha}\right)$ additive approximation of $\log Z(\lambda)$, where $\alpha=\left|\lambda-\lambda_{0}\right| / r<1$, and $n$ is the degree of $Z$ as a polynomial in $\lambda$. We note that Barvinok's approach may be seen as an algorithmic counterpart of the traditional view of phase transitions in statistical physics in terms of analyticity of $\log Z$ [55].

To apply this approach in the case of the ferromagnetic Ising model, we may appeal to the famous Lee-Yang theorem of the 1950s [29], which establishes that the zeros of $Z(\lambda)$ all lie on the unit circle in the complex plane. This allows us to take $\lambda_{0}=0$ and $r=1$ in the previous paragraph, and thus approximate $Z(\lambda)$ at any point $\lambda$ satisfying $|\lambda|<1$. This extends to all $\lambda$ with $|\lambda| \neq 1$ via the fact that $Z(\lambda)=\lambda^{n} Z\left(\frac{1}{\lambda}\right)$.

Remark. We note that the case $|\lambda|=1$ is likely to require additional ideas because it is known that there exist bounded degree graphs (namely $\Delta$-ary trees) for which the partition function $Z_{G}^{\beta}(\lambda)$ has complex zeros within distance $O(1 / n)$ of $\lambda=1$, where $n$ is the size of the graph. In fact, the zeros are even known to become dense on the whole unit circle as $n$ increases to infinity $[4,5]$. This precludes the possibility of efficiently carrying out the analytic continuation procedure for $\log Z$ to arbitrary points on the unit circle, and to the point $\lambda=1$ in particular.

Converting the above approach into an algorithm requires computing the first $k$ coefficients in the Taylor expansion of $\log Z$ around $\lambda_{0}$. Barvinok showed that this computation can in turn be reduced to computing the $O(k)$ lowest-degree coefficients of the partition function itself. In the case of the Ising model, computing $k$ such coefficients is roughly analogous to computing $k$-wise correlations between the vertex spins, and doing so naively on a graph of $n$ vertices requires time $\Omega\left(n^{k}\right)$. Until recently, no better ways to perform this calculation were known and hence approximation algorithms using this approach typically required quasi-polynomial time ${ }^{3}$ in order to achieve a $(1 \pm 1 / \operatorname{poly}(n))$-factor multiplicative approximation of $Z$ (equivalently, a $1 / \operatorname{poly}(n)$ additive approximation of $\log Z$ ), since this requires the Taylor series for $\log Z$ to be evaluated to $k=\Omega(\log n)$ terms $[7,9,10]$.

Recently, Patel and Regts [41] proposed a way to get around this barrier for several classes of partition functions. Their method is based on writing the coefficients in the Taylor series of $\log Z$ as linear combinations of counts of connected induced subgraphs of size up to $\Theta(\log n)$. This is already promising, since the number of connected induced subgraphs of size $O(\log n)$ of a graph $G$ of maximum degree $\Delta$ is polynomial in the size of $G$ when $\Delta$ is a fixed constant. Further, the count of induced copies of such a subgraph can also be computed in time polynomial in the size of $G$ [15]. Patel and Regts built on these tools to show a way to compute the $\Theta(\log n)$ Taylor coefficients of $\log Z$ needed in Barvinok's approach for several families of partition functions, for some of which correlation decay based algorithms are still not known.

Unfortunately, for the case of the Ising model, it is not clear how to write the Taylor coefficients in terms of induced subgraph counts. Indeed, in their paper [41, Theorem 1.4], Patel and Regts apply their method to the Ising model viewed as a polynomial in $\beta$ rather than $\lambda$, which allows them to use subgraph counts. However, this prevents them from using the Lee-Yang theorem, and they are consequently able to establish only a small zero-free region. As a result, they can handle only the zero-field "high-temperature" regime (where in fact the correlation decay property also holds), specifically the regime $|\beta-1| \leq 0.34 / \Delta$ and $\lambda=1$.

In this paper, we instead propose a generalization of the framework of Patel and Regts to labelled hypergraphs via objects that we call insects. In the special case of graphs, an insect can be seen as a graph that includes edges to additional boundary vertices: we refer to Section 3.1 for precise definitions. Using the appropriate notions for counting induced sub-insects, we are then able to write the coefficients arising in the Taylor expansion of $\log Z$ for the Ising model in terms of induced sub-insect counts, and derive from there algorithms for computing $\Omega(\log n)$ such coefficients in polynomial time in graphs of bounded degree. This establishes Theorem 1.1. We note that if one is only interested in deriving Theorem 1.1, then this can also be done using the notion of fragments, developed by Patel and Regts [41] in the different context of edge coloring models, which turns out to be a special case of our notion of insects.

\footnotetext{
${ }^{3}$ A quasi-polynomial time algorithm is one which runs in time $\exp \left\{O\left((\log n)^{c}\right)\right\}$ for some constant $c>1$.
} 
Our framework of insects, however, also allows us to extend the above approach to edge-dependent activities and, more significantly, to the much more general setting where $G$ is a hypergraph, as studied, for example, in the classical work of Suzuki and Fisher [53], and also more recently in the literature on approximate counting [20,32,50]. In a hypergraph of edge size $k \geq 3$, the pairwise interactions in the standard Ising model are replaced by higher-order interactions of order $k$. We note that the JerrumSinclair MCMC approach [27] apparently does not extend to hypergraphs, and there is currently no known polynomial time approximation algorithm (even randomized) for a wide range of $\beta$ in this setting. For a hypergraph $H=(V, E)$, configurations are again assignments of spins to the vertices $V$ and the partition function $Z_{H}^{\beta}(\lambda)$ is defined exactly as in (1), where the cut $E(S, \bar{S})$ consists of those hyperedges with at least one vertex in each of $S$ and $\bar{S}$. The computation of coefficients via insects carries through as before, but the missing ingredient is an extension of the Lee-Yang theorem to hypergraphs. Suzuki and Fisher [53] prove a weak version of the Lee-Yang theorem for hypergraphs (see Theorem 4.3 in section 4), which we are able to strengthen to obtain the following optimal statement, which is of independent interest:

Theorem 1.2. Let $H=(V, E)$ be a hypergraph with maximum hyperedge size $k \geq 3$. Then all the zeros of the Ising model partition function $Z_{H}^{\beta}(\lambda)$ lie on the unit circle if the edge activity $\beta$ lies in the range $-\frac{1}{2^{k-1}-1} \leq \beta \leq \frac{1}{2^{k-1} \cos ^{k-1}\left(\frac{\pi}{k-1}\right)+1}$. Further, when $\beta \neq 1$ does not lie in this range, there exists a hypergraph $H$ with maximum hypergraph edge size at most $k$ such that the zeros of the Ising partition function $Z_{H}^{\beta}(\lambda)$ of $H$ do not lie on the unit circle.

Remark. Once again, we can allow edge-dependent activities $\beta_{e}$ provided all of them lie in the range stipulated above. This extension also applies to Theorem 1.3 below.

Note that the classical Lee-Yang theorem (for the graph case $k=2$ ) establishes this property for $0 \leq \beta \leq 1$ (the ferromagnetic regime). Our proof of Theorem 1.2 follows along the lines of Asano's inductive proof of the Lee-Yang theorem [3], but we need to carefully analyze the base case (where $H$ consists of a single hyperedge) in order to obtain the above bounds on $\beta$. The optimality of the range of $\beta$ in our result follows essentially from the fact that our analysis of the base case is tight. For a detailed comparison with the Suzuki-Fisher theorem, see the Remark following Corollary 4.5.

Combining Theorem 1.2 with our earlier algorithmic approach immediately yields the following generalization of Theorem 1.1 to hypergraphs.

Theorem 1.3. Fix any $\Delta>0$ and $k \geq 3$. There is an FPTAS for the Ising partition function $Z_{H}^{\beta}(\lambda)$ in all hypergraphs $H$ of maximum degree $\Delta$ and maximum edge size $k$, for all edge activities $\beta$ in the range of Theorem 1.2 and all vertex activities $|\lambda| \neq 1$.

Finally, we extend our results to general ferromagnetic two-spin systems on hypergraphs, again as studied in [53]. A two-spin system on a hypergraph $H=(V, E)$ is specified by hyperedge activities $\varphi_{e}:\{+,-\}^{|e|} \rightarrow \mathbb{C}$ for $e \in E$, and a vertex activity $\psi:\{+,-\} \rightarrow \mathbb{C}$. (Note that we treat each hyperedge $e$ as a set of vertices.) Then the partition function is defined as:

$$
Z_{H}^{\varphi, \psi}:=\sum_{\boldsymbol{\sigma}: V \rightarrow\{+,-\}} \prod_{e \in E} \varphi_{e}\left(\left.\boldsymbol{\sigma}\right|_{e}\right) \prod_{v \in V} \psi(\boldsymbol{\sigma}(v))
$$

Without loss of generality, we will henceforth assume that $\varphi_{e}(-, \cdots,-)=1$, and that $\psi(-)=1$, $\psi(+)=\lambda$. We can then write the partition function as

$$
Z_{H}^{\varphi}(\lambda)=\sum_{\boldsymbol{\sigma}: V \rightarrow\{+,-\}} \prod_{e \in E} \varphi_{e}\left(\left.\boldsymbol{\sigma}\right|_{e}\right) \lambda^{|\{v: \boldsymbol{\sigma}(v)=+\}|} .
$$

We call a hypergraph two-spin system symmetric if $\varphi_{e}(\boldsymbol{\sigma})=\overline{\varphi_{e}(-\boldsymbol{\sigma})}$. Suzuki and Fisher [53] proved a Lee-Yang theorem for symmetric hypergraph two-spin systems (which is weaker than our Theorem 1.2 
above when specialized to the Ising model). Combining this with our general algorithmic approach yields our final result:

Theorem 1.4. Fix any $\Delta>0$ and $k \geq 2$ and a family of symmetric edge activities $\varphi=\left\{\varphi_{e}\right\}$ satisfying $\left|\varphi_{e}(+, \cdots,+)\right| \geq \frac{1}{4} \sum_{\boldsymbol{\sigma} \in\{+,-\}^{V}}\left|\varphi_{e}(\boldsymbol{\sigma})\right|$. Then there exists an FPTAS for the partition function $Z_{H}^{\varphi}(\lambda)$ of the corresponding symmetric hypergraph two-spin system in all hypergraphs $H$ of maximum degree $\Delta$ and maximum edge size $k$ for all vertex activities $\lambda \in \mathbb{C}$ such that $|\lambda| \neq 1$.

The remainder of the paper is organized as follows. In section 2, we spell out Barvinok's approach to approximating partition functions using Taylor series. Section 3 introduces the notion of insects and shows how to use them to efficiently compute the lowest-degree coefficients of the partition function in the general context of hypergraphs; as discussed above, this machinery applied to graphs, in conjunction with the Lee-Yang theorem, implies Theorem 1.1. Finally, in section 4 we prove our extension of the Lee-Yang theorem to the hypergraph Ising model (Theorem 1.2), and then use it and the Suzuki-Fisher theorem to prove our algorithmic results for hypergraphs, Theorems 1.3 and 1.4.

1.1. Related work. The problem of computing partition functions has been widely studied, not only in statistical physics but also in combinatorics, because the partition function is often a generating function for combinatorial objects (cuts, in the case of the Ising model). There has been much progress on dichotomy theorems, which attempt to completely classify these problems as being either \#P-hard or computable (exactly) in polynomial time (see, e.g., [16,21]).

Since the problems are in fact \#P-hard in most cases, algorithmic interest has focused largely on approximation, motivated also by the general observation that approximating the partition function is polynomial time equivalent to sampling (approximately) from the underlying Gibbs distribution [28]. In fact, most early approximation algorithms exploited this connection, and gave fully-polynomial randomized approximation schemes (FPRAS) for the partition function using Markov chain Monte Carlo (MCMC) samplers for the Gibbs distribution. In particular, for the ferromagnetic Ising model, the MCMC-based algorithm of Jerrum and Sinclair [27] is valid for all positive real values of $\lambda$ and for all graphs, irrespective of their vertex degrees. (For the connection with random sampling in this case, see [42].) This was later extended to ferromagnetic two-spin systems by Goldberg, Jerrum and Paterson [22]. Similar techniques have been applied recently to the related random-cluster model by Guo and Jerrum [23].

Much detailed work has been done on MCMC for Ising spin configurations for several important classes of graphs, including two-dimensional lattices (e.g., [33,37,38]), random graphs and graphs of bounded degree (e.g., [40]), the complete graph (e.g., [31]) and trees (e.g., [11,39]); we do not attempt to give a comprehensive summary of this line of work here.

In the anti-ferromagnetic regime $(\beta>1)$, deterministic approximation algorithms based on correlation decay have been remarkably successful for graphs of bounded degree. Specifically, for any fixed integer $\Delta \geq 3$, techniques of Weitz [54] give a (deterministic) FPTAS for the anti-ferromagnetic Ising partition function on graphs of maximum degree $\Delta$ throughout a region $R_{\Delta}$ in the $(\beta, \lambda)$ plane (corresponding to the regime of uniqueness of the Gibbs measure on the $\Delta$-regular tree) [30,47]. A complementary result of Sly and Sun [49] (see also [19]) shows that the problem is NP-hard outside $R_{\Delta}$. In contrast, no MCMC based algorithms are known to provide an FPRAS for the anti-ferromagnetic Ising partition function throughout $R_{\Delta}$. More recently, correlation decay techniques have been extended to obtain deterministic approximation algorithms for the anti-ferromagnetic Ising partition function on hypergraphs over a range of parameters [32], as well as to several other problems not related to the Ising model. In the ferromagnetic setting, however, for reasons mentioned earlier, correlation decay techniques have had more limited success: Zhang, Liang and Bai [56] handle only the "high-temperature" regime of the Ising model, while the recent results for ferromagnetic two-spin systems of Guo and $\mathrm{Lu}$ [24] do not apply to the case of the Ising model.

In a parallel line of work, Barvinok initiated the study of Taylor approximation of the logarithm of the partition function, which led to quasipolynomial time approximation algorithms for a variety of counting 
problems $[6,7,9,10]$. More recently, Patel and Regts [41] showed that for several models that can be written as induced subgraph sums, one can actually obtain an FPTAS from this approach. In particular, for problems such as counting independent sets with negative (or, more generally, complex valued) activities on bounded degree graphs, they were able to match the range of applicability of existing algorithms based on correlation decay, and were also able to extend the approach to Tutte polynomials and edge-coloring models (also known as Holant problems) where little is known about correlation decay.

The Lee-Yang program was initiated by Lee and Yang [55] in connection with the analysis of phase transitions. By proving the famous Lee-Yang circle theorem for the ferromagnetic Ising model [29], they were able to conclude that there can be at most one phase transition for the model. Asano [3] extended the Lee-Yang theorem to the Heisenberg model, and provided a simpler proof. Asano's work was generalized further by Suzuki and Fisher [53], while Sinclair and Srivastava [46] studied the multiplicity of Lee-Yang zeros. A complete characterization of Lee-Yang polynomials that are independent of the "temperature" of the model was recently obtained by Ruelle [43]. The study of Lee-Yang type theorems for other statistical physics models has also generated beautiful connections with other areas of mathematics. For example, Shearer [45] and Scott and Sokal [44] established the close connection between the location of the zeros of the independence polynomial and the Lovász Local Lemma, while the study of the zeros of generalizations of the matching polynomial was used in the recent celebrated work of Marcus, Spielman and Srivastava on the existence of Ramanujan graphs [35]. Such Lee-Yang type theorems are exemplars of the more general stability theory of polynomials $[12,13]$, a field of study that has had numerous recent applications to theoretical computer science and combinatorics (see, e.g., $[1,2,14,35,36,46,52]$ ).

\section{APPROXIMATION OF THE LOG-PARTITION FUNCTION BY TAYLOR SERIES}

In this section we present an approach due to Barvinok [7] for approximating the partition function of a physical system by truncating the Taylor series of its logarithm, as discussed in the introduction. We will work in our most general setting of symmetric two-spin systems on hypergraphs, which of course includes the Ising model (on graphs or hypergraphs) as a special case. As in (2), such a system has partition function

$$
Z_{H}^{\varphi}(\lambda)=\sum_{\boldsymbol{\sigma}: V \rightarrow\{+,-\}} \prod_{e \in E} \varphi_{e}\left(\left.\boldsymbol{\sigma}\right|_{e}\right) \lambda^{|\{v: \boldsymbol{\sigma}(v)=+\}|} .
$$

Our goal is a FPTAS for $Z_{H}^{\varphi}(\lambda)$, i.e., a deterministic algorithm that, given as input $H,\left\{\varphi_{e}\right\}, \lambda$ with $|\lambda| \neq 1$ and $\varepsilon \in(0,1]$, runs in time polynomial in $n=|H|$ and $\varepsilon^{-1}$ and outputs a $(1 \pm \varepsilon)$-multiplicative approximation of $Z_{H}^{\varphi}(\lambda)$, i.e., a number $\hat{Z}$ satisfying

$$
\left|\hat{Z}-Z_{H}^{\varphi}(\lambda)\right| \leq \varepsilon\left|Z_{H}^{\varphi}(\lambda)\right|
$$

(Note that in our setting $\hat{Z}$ and $Z_{H}^{\varphi}(\lambda)$ may be complex numbers.) By the symmetry $\varphi_{e}(\boldsymbol{\sigma})=\overline{\varphi_{e}(-\boldsymbol{\sigma})}$, we also have $Z^{\varphi}(\lambda)=\lambda^{n} Z^{\bar{\varphi}}\left(\frac{1}{\lambda}\right)$, so that without loss of generality we may assume $|\lambda|<1$.

For fixed $H$ and (hyper)edge activities $\varphi$, we will write $Z(\lambda)=Z_{H}^{\varphi}(\lambda)$ for short. Letting $f(\lambda)=\log Z(\lambda)$, using the Taylor expansion around $\lambda=0$ we get

$$
f(\lambda)=\sum_{j=0}^{\infty} f^{(j)}(0) \cdot \frac{\lambda^{j}}{j !},
$$

where $f(0)=\log Z(0)=0$. Note that $Z=\exp (f)$, and thus an additive error in $f$ translates to a multiplicative error in $Z$. More precisely, given $\varepsilon \leq 1 / 4$, and $f, \tilde{f} \in \mathbb{C}$ such that $|f-\widetilde{f}| \leq \varepsilon$, we have

$$
|\exp (\widetilde{f})-\exp (f)|=|\exp (\widetilde{f}-f)-1| \times|\exp (f)| \leq 4 \varepsilon|\exp (f)|,
$$

where the last inequality, valid for $\varepsilon \leq 1 / 4$, follows by elementary complex analysis. In other words, to achieve a multiplicative approximation of $Z$ within a factor $1 \pm \varepsilon$, as required by a FPTAS, it suffices to obtain an additive approximation of $f$ within $\varepsilon / 4$. 
To get an additive approximation of $f$, we use the first $m$ terms in the Taylor expansion. Specifically, we compute $f_{m}(\lambda):=\sum_{j=0}^{m} f^{(j)}(0) \cdot \frac{\lambda^{j}}{j !}$. We show next how to compute the derivatives $f^{(j)}(0)$ from the derivatives of $Z$ itself (which are more readily accessible).

To compute $f^{(j)}(0)$, note that $f^{\prime}(\lambda)=\frac{1}{Z(\lambda)} \frac{\mathrm{d} Z(\lambda)}{\mathrm{d} \lambda}$, or $\frac{\mathrm{d} Z(\lambda)}{\mathrm{d} \lambda}=f^{\prime}(\lambda) Z(\lambda)$. Thus for any $m \geq 1$,

$$
\frac{\mathrm{d}^{m}}{\mathrm{~d} \lambda^{m}} Z(\lambda)=\sum_{j=0}^{m-1}\left(\begin{array}{c}
m-1 \\
j
\end{array}\right) \frac{\mathrm{d}^{j}}{\mathrm{~d} \lambda^{j}} Z(\lambda) \cdot \frac{\mathrm{d}^{m-j}}{\mathrm{~d} \lambda^{m-j}} f(\lambda) .
$$

Given $\left.\frac{\mathrm{d}^{j}}{\mathrm{~d} \lambda^{j}} Z(\lambda)\right|_{\lambda=0}$ for $j=0, \cdots, m$, eq. (5) is a triangular system of linear equations in $\left\{f^{(j)}(0)\right\}_{j=1}^{m}$ of representation length poly $(m)$, and is non-degenerate since $Z(0)=1$; hence it can be solved in poly $(m)$ time.

We can now specify the algorithm: first compute $\left\{\left.\frac{\mathrm{d}^{j}}{\mathrm{~d} \lambda^{j}} Z(\lambda)\right|_{\lambda=0}\right\}_{j=0}^{m}$; next, use the system in eq. (5) to solve for $\left\{f^{(j)}(0)\right\}_{j=1}^{m}$; and finally, compute and ouput the approximation $f_{m}(\lambda)$.

To quantify the approximation error in this algorithm, we need to study the locations of the complex roots $r_{1}, \cdots, r_{n}$ of $Z$. Throughout this paper, we will be using (some variant of) the Lee-Yang theorem to argue that, for the range of interactions $\varphi$ we are interested in, the roots $r_{i}$ all lie on the unit circle in the complex plane, i.e., $\left|r_{i}\right|=1$ for all $i$. Note that since we are assuming that $\varphi_{e}(-, \cdots,-)=1$, the constant term $\prod_{i=1}^{n}\left(-r_{i}\right)$ of $Z(\lambda)$ is 1 , and hence we have $Z(\lambda)=\prod_{i}\left(1-\frac{\lambda}{r_{i}}\right)$. The log partition function can then be written as

$$
f(\lambda)=\log Z(\lambda)=\sum_{i=1}^{n} \log \left(1-\frac{\lambda}{r_{i}}\right)=-\sum_{i=1}^{n} \sum_{j=1}^{\infty} \frac{1}{j}\left(\frac{\lambda}{r_{i}}\right)^{j} .
$$

Note that due to the uniqueness of the Taylor expansion of meromorphic functions, the two power series expansions of $f(\lambda)$ in eqs. (4) and (6) are identical in the domain of their convergence. Denoting the first $m$ terms of the above expansion by $f_{m}(\lambda)=-\sum_{i=1}^{n} \sum_{j=1}^{m} \frac{1}{j}\left(\frac{\lambda}{r_{i}}\right)^{j}$, the error due to truncation is bounded by

$$
\left|f(\lambda)-f_{m}(\lambda)\right| \leq n \sum_{j=m+1}^{\infty} \frac{|\lambda|^{j}}{j} \leq \frac{n|\lambda|^{m+1}}{(m+1)(1-|\lambda|)},
$$

recalling that by symmetry we are assuming $|\lambda|<1$. Thus to get within $\varepsilon / 4$ additive error, it suffices to take $m \geq \frac{1}{\log (1 /|\lambda|)}\left(\log \left(\frac{4 n}{\varepsilon}\right)+\log \left(\frac{1}{1-|\lambda|}\right)\right)$. The following result summarizes our discussion so far.

Lemma 2.1. Given $\varepsilon \in(0,1), m \geq \frac{1}{\log (1 /|\lambda|)}\left(\log \left(\frac{4 n}{\varepsilon}\right)+\log \left(\frac{1}{1-|\lambda|}\right)\right)$, and the values $\left\{\left.\frac{\mathrm{d}^{j}}{\mathrm{~d} \lambda^{j}} Z(\lambda)\right|_{\lambda=0}\right\}_{j=0}^{m}$, $f_{m}(\lambda)$ can be computed in time poly $(n / \varepsilon)$. Moreover, if the Lee-Yang theorem holds for the partition function $Z(\lambda)$, then $\left|f_{m}(\lambda)-f(\lambda)\right|<\varepsilon / 4$, and thus $\exp \left(f_{m}(\lambda)\right)$ approximates $Z(\lambda)$ within a multiplicative factor $1 \pm \varepsilon$.

The missing ingredient in turning Lemma 2.1 into an FPTAS is the computation of the derivatives $\left.\frac{\mathrm{d}^{j}}{\mathrm{~d} \lambda^{j}} Z(\lambda)\right|_{\lambda=0}$ for $1 \leq j \leq m$, which themselves are just multiples of the $m+1$ lowest-degree coefficients of $Z$. Computing these values naively using the definition of $Z(\lambda)$ requires $n^{\Omega(m)}$ time. Since $m$ is required to be of order $\Omega(\log (n / \varepsilon))$, this results in only a quasi-polynomial time algorithm. In the next section, we show how to compute these values in polynomial time when $H$ is a hypergraph of bounded degree and bounded hyperedge size, which when combined with Lemma 2.1 gives an FPTAS.

\section{COMPUTING COEFFICIENTS VIA INSECTS}

As discussed in the introduction, Patel and Regts [41] recently introduced a technique for efficiently computing the low-degree coefficients of a partition function using induced subgraph counts. In this section we introduce the notion of sub-insect counts, and show how it allows the Patel-Regts framework to be 
adapted to any hypergraph two-spin system with vertex activities (including the Ising model with vertex activities as a special case). We will align our notation with [41] as much as possible. From now on, unless otherwise stated, we will use $G$ to denote a hypergraph. Recall from the introduction the partition function of a two-spin system on a hypergraph $G=(V, E)$ :

$$
Z_{G}^{\varphi}(\lambda)=\sum_{\boldsymbol{\sigma}: V \rightarrow\{+,-\}} \prod_{e \in E} \varphi_{e}\left(\left.\boldsymbol{\sigma}\right|_{e}\right) \lambda^{|\{v: \boldsymbol{\sigma}(v)=+\}|} .
$$

Due to the normalization $\varphi_{e}(-, \cdots,-)=1$, each term in the summation depends only on the set $S=\{v: \boldsymbol{\sigma}(v)=+\}$ and the labelled induced sub-hypergraph $(S \cup \partial S, E[S] \cup E(S, \bar{S}))$, where $E[S]$ is the set of edges within $S, \partial S$ is the boundary of $S$ defined as $\partial S:=\bigcup_{v \in S} N_{G}(v) \backslash S$, and $N_{G}(v)$ is the set of vertices adjacent to the vertex $v$ in $G$. This fact motivates the induced sub-structures we will consider.

Let $\sigma^{S}$ be the configuration where the set of vertices assigned +-spins is $S$, that is, $\boldsymbol{\sigma}^{S}(v)=+$ for $v \in S$ and $\boldsymbol{\sigma}^{S}(v)=-$ otherwise. We will also write $\varphi_{e}(S):=\varphi_{e}\left(\left.\boldsymbol{\sigma}^{S}\right|_{e}\right)$ for simplicity. Thus the partition function can be written

$$
Z_{G}^{\varphi}(\lambda)=\sum_{S \subseteq V} \prod_{e: e \cap S \neq \emptyset} \varphi_{e}(S) \lambda^{|S|} .
$$

We start with the standard factorization of the partition function in terms of its complex zeros $r_{1}, \ldots, r_{n}$, where $n=|V|$. As explained in the paragraph preceding eq. (6), the assumption $\varphi_{e}(-, \cdots,-)=1$ allows one to write the partition function as

$$
Z_{G}^{\varphi}(\lambda)=\prod_{j=1}^{n}\left(1-\lambda / r_{j}\right)=\sum_{i=0}^{n}(-1)^{i} e_{i}(G) \lambda^{i},
$$

where $e_{i}(G)$ is the elementary symmetric polynomial of degree $i$ evaluated at $\left(\frac{1}{r_{1}}, \cdots, \frac{1}{r_{n}}\right)$.

On the other hand, we can also express the coefficients $e_{i}(G)$ combinatorially using the definition of the partition function:

$$
e_{i}(G)=(-1)^{i} \sum_{\substack{S \subseteq V \\|S|=i}} \prod_{e: e \cap S \neq \emptyset} \varphi_{e}(S)
$$

Once we have computed the first $m$ coefficients of $Z$ (i.e., the values $e_{i}(G)$ for $i=1, \cdots, m$ ), where $m=\Omega\left(\frac{\log (n / \varepsilon)-\log (1-|\lambda|)}{\log (1 /|\lambda|)}\right)$, we can use Lemma 2.1 to obtain an FPTAS as claimed in Theorems 1.1, 1.3 and 1.4. The main result in this section will be an algorithm for computing these coefficients $e_{i}(G)$ :

Theorem 3.1. Fix $k, \Delta \in \mathbb{N}$ and $C>0$. There exists a deterministic $\operatorname{poly}(n / \varepsilon)$-time algorithm that, given any $n$-vertex hypergraph $G$ of maximum degree $\Delta$ and maximum hyperedge size $k$, and any $\varepsilon \in(0,1)$, computes the coefficients $e_{i}(G)$ for $i=1, \cdots, m$, where $m=\lceil C \log (n / \varepsilon)\rceil$.

3.1. Insects in a hypergraph. To take advantage of the fact that each term in eq. (7) only depends on the set $S$ and the induced sub-hypergraph $(S \cup \partial S, E[S] \cup E(S, \bar{S}))$, we define the following structure.

Definition 3.2. Given a vertex set $S$ and a set $E$ of hyperedges, $H=(S, E)$ is called an insect if for all $e \in E, e \cap S \neq \emptyset$. The set $S$ is called the label set of the insect $H$ and the set $B(H):=\left(\bigcup_{e \in E} e\right) \backslash S$ is called the boundary set.

Given an insect $H$, we use the notation $\underline{V}(H)$ for its label set. The size $|H|$ of the insect $H$ is defined to be $|\underline{V}(H)|$. An insect $H=(S, E)$ is said to be connected if the hypergraph $(S,\{e \cap S \mid e \in E\})$ is connected. It is said to be disconnected otherwise. In the latter case, there exists a partition of $S$ into non-empty sets $S_{1}, S_{2}$, and a partition of $E$ into sets $E_{1}$ and $E_{2}$, such that $\left(S_{i}, E_{i}\right)$ are insects for $i=1,2$, and the sets $S_{2} \cap B\left(H_{1}\right)$ and $S_{1} \cap B\left(H_{2}\right)$ are empty. In this case, we write $H=H_{1} \uplus H_{2}$, and say that the insects $H_{1}$ and $H_{2}$ are disjoint. (Note that disjoint insects may share boundary vertices.) 
Remark. Note that a hypergraph $G=(V, E)$ can itself be viewed as an insect. However, as is clear from the definition, not all insects are hypergraphs.

In order to exploit the structure of the terms in eq. (7) alluded to above, we now define the notion of an induced sub-insect of an insect. Given an insect $H=(S, E)$ and a subset $S^{\prime}$ of $S$, we define the induced sub-insect $H^{+}\left[S^{\prime}\right]$ as $\left(S^{\prime},\left\{e \in E \mid e \cap S^{\prime} \neq \emptyset\right\}\right)$. Further, we say that an insect $H$ is an induced sub-insect of an insect $G$, denoted $H \hookrightarrow G$, if there is a set $S \subseteq \underline{V}(G)$ such that $G^{+}[S]=H$.

3.2. Weighted sub-insect counts. Just as graph invariants may be expressed as sums over induced subgraph counts, we will consider weighted sub-insect counts of the form $f(G)=\sum_{S \subset V(G)} a_{G^{+}[S]}$ and the functions $f$ expressible in this way. Here $G$ is any insect, and the coefficients $a_{H}$ depend only on $H$, not on $G$.

Let $\mathcal{G}_{t}^{\Delta, k}$ be the set of insects up to size $t$, with maximum degree $\Delta$ and maximum hyperedge size $k$. Note that since insects are labelled, this is an infinite set. We will fix $\Delta$ and $k$ throughout, and write $\mathcal{G}:=\bigcup_{t \geq 1} \mathcal{G}_{t}^{\Delta, k}$. Let $\mathbf{1}[H \hookrightarrow G]$ be the indicator that $H$ is an induced sub-insect of $G$, that is,

$$
\mathbf{1}[H \hookrightarrow G]=1 \text { if there is a set } S \subseteq \underline{V}(G) \text { such that } G^{+}[S]=H \text {, and } 0 \text { otherwise. }
$$

A weighted sub-insect count $f(G)$ of the form considered above can then also be written as $f(G)=$ $\sum_{H \in \mathcal{G}} a_{H} \cdot \mathbf{1}[H \hookrightarrow G]$. This alternative notation helps simplify the presentation of some of the combinatorial arguments below. Note that even though $\mathcal{G}$ is infinite, the above sum has only finitely many non-zero terms for any finite insect $G$. Further, as insects are labelled, $f(G)$ may also depend on the labelling of $G$, unlike a graph invariant where isomorphic copies of a graph yield the same value.

A weighted sub-insect count $f$ is said to be additive if, given any two disjoint insects $G_{1}$ and $G_{2}$, $f\left(G_{1} \uplus G_{2}\right)=f\left(G_{1}\right)+f\left(G_{2}\right)$. An argument due to Csikvári and Frenkel [17], also employed in the case of graph invariants by Patel and Regts [41], can then be adapted to give the following:

Lemma 3.3. Let $f$ be a weighted sub-insect count, so that $f$ may be written as

$$
f(G)=\sum_{H \in \mathcal{G}} a_{H} \cdot \mathbf{1}[H \hookrightarrow G] .
$$

Then $f$ is additive if and only if $a_{H}=0$ for all insects $H$ that are disconnected.

Proof. When $H$ is connected, we have $\mathbf{1}\left[H \hookrightarrow G_{1} \uplus G_{2}\right]=\mathbf{1}\left[H \hookrightarrow G_{1}\right]+\mathbf{1}\left[H \hookrightarrow G_{2}\right]$; thus $f$ given in the above form is additive if $a_{H^{\prime}}=0$ for all $H^{\prime}$ that are not connected.

Conversely, suppose $f$ is additive. By the last paragraph, we can assume without loss of generality that the sequence $a_{H}$ is supported on disconnected insects (by subtracting the component of $f$ supported on connected $H$ ). We now show that for such an $f, a_{H}$ must be 0 for all disconnected $H$ as well.

For if not, let $H$ be a (necessarily disconnected) insect of smallest size for which $a_{H} \neq 0$. Since $a_{J}=0$ for all insects $J$ with $|J|<|H|$, we must have $f(J)=0$ for all such insects. Also, since $H$ is disconnected, there exist non-empty insects $H_{1}$ and $H_{2}$ such that $H=H_{1} \uplus H_{2}$. By additivity, we then have $f(H)=f\left(H_{1}\right)+f\left(H_{2}\right)=0$, where the last equality follows since both $\left|H_{1}\right|,\left|H_{2}\right|$ are strictly smaller than $|H|$. On the other hand, since $H$ is an insect with the smallest possible number of vertices such that $a_{H} \neq 0$, we also have $f(H)=a_{H} \mathbf{1}[H \hookrightarrow H]=a_{H}$. This implies $a_{H}=0$, which is a contradiction. Hence we must have $a_{H}=0$ for all disconnected $H$.

The next lemma implies that the product of weighted sub-insect counts can also be expressed as a weighted sub-insect count. We begin with a definition.

Definition 3.4. An insect $H_{1}=\left(S_{1}, E_{1}\right)$ is compatible with another insect $H_{2}=\left(S_{2}, E_{2}\right)$ if the insect $H:=\left(S_{1} \cup S_{2}, E_{1} \cup E_{2}\right)$ satisfies $H^{+}\left[S_{1}\right]=H_{1}$ and $H^{+}\left[S_{2}\right]=H_{2}$.

Lemma 3.5. Let $H_{1}=\left(S_{1}, E_{1}\right), H_{2}=\left(S_{2}, E_{2}\right)$ be arbitrary insects. 
(i) If $H_{1}$ and $H_{2}$ are not compatible, then there is no insect $G$ such that $H_{1} \hookrightarrow G$ and $H_{2} \hookrightarrow G$. In other words, for every insect $G$,

$$
\mathbf{1}\left[H_{1} \hookrightarrow G\right] \mathbf{1}\left[H_{2} \hookrightarrow G\right]=0 .
$$

(ii) If $H_{1}$ and $H_{2}$ are compatible, then for every insect $G$,

$$
\mathbf{1}\left[H_{1} \hookrightarrow G\right] \mathbf{1}\left[H_{2} \hookrightarrow G\right]=\mathbf{1}[H \hookrightarrow G],
$$

where $H$ is the insect $\left(S_{1} \cup S_{2}, E_{1} \cup E_{2}\right)$, and satisfies $H^{+}\left[S_{i}\right]=H_{i}$ for $i=1,2$.

Proof. We start by making two observations. First, if $G^{+}\left[S_{1}\right]=H_{1}$ and $G^{+}\left[S_{2}\right]=H_{2}$ then $G^{+}\left[S_{1} \cup S_{2}\right]=$ $H=\left(S_{1} \cup S_{2}, E_{1} \cup E_{2}\right)$. Second, if $T \subseteq S \subseteq V$ and $G_{1}:=G^{+}[S]$ then $G_{1}^{+}[T]=G^{+}[T]$.

Suppose first that $H_{1}$ and $H_{2}$ are not compatible. Suppose, for the sake of contradiction, that there exists an insect $G$ such that $G^{+}\left[S_{i}\right]=H_{i}$ for $i=1,2$. Then, from the first observation above we have $G^{+}\left[S_{1} \cup S_{2}\right]=H=\left(S_{1} \cup S_{2}, E_{1} \cup E_{2}\right)$, while from the second observation we have $H^{+}\left[S_{i}\right]=G^{+}\left[S_{i}\right]=$ $H_{i}$ for $i=1,2$. This contradicts the assumption that $H_{1}$ and $H_{2}$ are incompatible. Thus, we must have $\mathbf{1}\left[H_{1} \hookrightarrow G\right] \mathbf{1}\left[H_{2} \hookrightarrow G\right]=0$ for every $G$, proving part (i).

Now suppose that $H_{1}$ and $H_{2}$ are compatible. As seen above, $G^{+}\left[S_{i}\right]=H_{i}$ for $i=1,2$ implies that $G^{+}\left[S_{1} \cup S_{2}\right]=H$. On the other hand, if $G^{+}\left[S_{1} \cup S_{2}\right]=H$, then by the compatibility of $H_{1}$ and $H_{2}$, and the second observation above, $G^{+}\left[S_{i}\right]=H^{+}\left[S_{i}\right]=H_{i}$ for $i=1,2$. This proves part (ii) of the lemma.

An immediate corollary of the above lemma is that a product of weighted sub-insect counts is also a sub-insect count supported on slightly larger insects.

Corollary 3.6. If $f_{1}(G)=\sum_{H} a_{H} \cdot \mathbf{1}[H \hookrightarrow G]$ and $f_{2}(G)=\sum_{H} b_{H} \cdot \mathbf{1}[H \hookrightarrow G]$ are weighted sub-insect counts, then so is $g(G):=f_{1}(G) f_{2}(G)$. Moreover, if $f_{1}, f_{2}$ are supported on sub-insects of sizes $\leq t_{1}, t_{2}$ respectively (i.e., if $a_{H}=0$ when $|H|>t_{1}$ and $b_{H}=0$ when $|H|>t_{2}$ ), then $g$ is supported on sub-insects of size $\leq t_{1}+t_{2}$.

Proof. For compatible insects $H_{i}=\left(S_{i}, E_{i}\right)$ we denote by $H_{1} \cup H_{2}$ the insect $\left(S_{1} \cup S_{2}, E_{1} \cup E_{2}\right)$. Now, for any insect $G$ we have,

$$
\begin{aligned}
g(G) & =\sum_{H_{1}, H_{2}} a_{H_{1}} b_{H_{2}} \cdot \mathbf{1}\left[H_{1} \hookrightarrow G\right] \cdot \mathbf{1}\left[H_{2} \hookrightarrow G\right] \\
& =\sum_{H_{1}, H_{2} \text { compatible }} a_{H_{1}} b_{H_{2}} \cdot \mathbf{1}\left[H_{1} \cup H_{2} \hookrightarrow G\right] \\
& =\sum_{H} c_{H} \cdot \mathbf{1}[H \hookrightarrow G],
\end{aligned}
$$

where in the second line we have used Lemma 3.5, and where

$$
c_{H}:=\sum_{\substack{H_{1}, H_{2} \text { compatible } \\ H=H_{1} \cup H_{2}}} a_{H_{1}} b_{H_{2}} .
$$

Note that the number of non-zero terms in the definition of each $c_{H}$ is finite, and that $\left|H_{1} \cup H_{2}\right| \leq\left|H_{1}\right|+\left|H_{2}\right|$. This completes the proof.

3.3. Enumerating connected sub-insects. We observe next that $e_{i}(G)$, as defined in eq. (8), can be written as a weighted sub-insect count. Accordingly, we generalize eq. (8) to arbitrary insects $G$ of maximum degree $\Delta$ and hyperedge size $k$ as follows:

$$
e_{i}(G)=(-1)^{i} \sum_{\substack{S \subseteq V(G) \\|S|=i}} \prod_{e: e \cap S \neq \emptyset} \varphi_{e}(S)=\sum_{H \in \mathcal{G}_{i}^{\Delta, k}} \mu_{H} \cdot \mathbf{1}[H \hookrightarrow G],
$$


where $\mu_{H}:=(-1)^{i} \prod_{e: e \cap V(H) \neq \emptyset} \varphi_{e}(\underline{V}(H))$. Note that this definition coincides with eq. (8) when $G$ is a hypergraph, and also extends the definition of the partition function from hypergraphs to insects via the equation $Z_{G}(\lambda)=\sum_{i=0}^{|G|}(-1)^{i} e_{i}(G) \lambda^{i}$; when $G=(S, E)$ this definition is equivalent to that of the partition function on the hypergraph $(S \cup B(G), E)$, with the vertices in $B(G)$ set to '-'. This latter observation implies that when the insect $G$ is disconnected and $G=G_{1} \uplus G_{2}$, we have $Z_{G}(\lambda)=$ $Z_{G_{1}}(\lambda) Z_{G_{2}}(\lambda)$.

We now consider the computational properties of the above expansion. Note that each coefficient $\mu_{H}$ is readily computable in time poly $(|H|)$; however, as discussed in the introduction, the number of $H \in \mathcal{G}_{i}^{\Delta, k}$ such that $\mathbf{1}[H \hookrightarrow G] \neq 0$ is $\Omega\left(n^{i}\right)$, so that a naive computation of $e_{i}(G)$ using eq. (10) would be inefficient. To prove Theorem 3.1, we consider the set of connected insects, denoted by $\mathcal{C}_{i}^{\Delta, k}$, rather than $\mathcal{G}_{i}^{\Delta, k}$. We will show in this subsection that $\mathcal{C}_{i}^{\Delta, k}$ can be efficiently enumerated, and then in the following subsection reduce the above summation over $\mathcal{G}_{i}^{\Delta, k}$ to enumerations of $\mathcal{C}_{i}^{\Delta, k}$.

As in [41], we use the following calculation of Borgs et al. [15, Lemma 2.1 (c)].

Lemma 3.7. Let $G$ be a multigraph with maximum degree $\Delta$ (counting edge multiplicity) and let $v$ be a vertex of $G$. Then the number of subtrees of $G$ with $t$ vertices containing the vertex $v$ is at most $\frac{(e \Delta)^{t-1}}{2}$.

Proof. Consider the infinite rooted $\Delta$-ary tree $T_{\Delta}$. The number of subtrees with $t$ vertices starting from the root is $\frac{1}{t}\left(\begin{array}{c}t \Delta \\ t-1\end{array}\right)<\frac{(e \Delta)^{t-1}}{2}$. (See also [51, Theorem 5.3.10].) The proof is completed by observing that the set of $t$-vertex subtrees of $G$ containing vertex $v$ can be mapped injectively into subtrees of $T_{\Delta}$ containing the root.

Corollary 3.8. Let $G$ be a hypergraph with maximum degree $\Delta$ and maximum hyperedge size $k$, and let $v \in V(G)$. Then the number of connected induced sub-insects of $G$ of size $t$ whose label set contains the vertex $v$ is at most $\frac{(e \Delta k)^{t-1}}{2}$.

Proof. Consider the multigraph $H$ obtained by replacing every hyperedge of size $r$ in $G$ by an $r$-clique. For any connected induced sub-insect $A$ of $G$, the label set $\underline{V}(A)$ is connected in $H$. Now, for any two distinct connected induced sub-insects $A$ and $B$, let $S_{A}$ and $S_{B}$ be the sets of trees in $H$ that span the label sets $\underline{V}(A)$ and $\underline{V}(B)$ of $A$ and $B$ respectively. Since the label sets of $A$ and $B$ are different, we must have $S_{A} \cap S_{B}=\emptyset$. Thus the number of connected subtrees on $t$ vertices in $H$ which contain the vertex $v$ is an upper bound on the number of connected induced sub-insects in $G$ whose label set contains $v$.

Finally, in the multigraph $H$ the maximum degree is $\Delta k$, so by Lemma 3.7 the number of such subtrees is at most $\frac{(e \Delta k)^{t-1}}{2}$.

As a consequence we can efficiently enumerate all connected induced sub-insects of logarithmic size in a bounded degree graph. This follows from a similar reduction to a multigraph, applying [41, Lemma 3.4]. However, for the sake of completeness we also include a direct proof.

Lemma 3.9. For a hypergraph $G$ of maximum degree $\Delta$ and maximum hyperedge size $k$, there exists an algorithm that enumerates all connected induced sub-insects of size at most $t$ in $G$ and runs in time $\widetilde{O}\left(n t^{3}(e \Delta k)^{t+1}\right)$. Here $\widetilde{O}$ hides factors of the form $\operatorname{polylog}(n), \operatorname{polylog}(\Delta k)$ and $\operatorname{polylog}(t)$.

Proof. Let $\mathcal{T}_{t}$ be the set of $S \subseteq V(G)$ such that $|S| \leq t$ and $G^{+}[S]$ is connected. Note that given $S \in \mathcal{T}_{t}$, $G^{+}[S]$ will be a sub-insect of size $t$, and this clearly enumerates all of them. Also, by Corollary 3.8, $\left|\mathcal{T}_{t}\right| \leq O\left(n(e \Delta k)^{t}\right)$. Thus it remains to give an algorithm to construct $\mathcal{T}_{t}$ in about the same amount of time.

We construct $\mathcal{T}_{t}$ inductively. For $t=1, \mathcal{T}_{1}:=V(G)$. Then given $\mathcal{T}_{t-1}$, define the multiset

$$
\mathcal{T}_{t}^{*}:=\mathcal{T}_{t-1} \cup\left\{S \cup\{v\}: S \in \mathcal{T}_{t-1} \text { and } v \in N_{G}(S) \backslash S\right\} .
$$

Since $\left|N_{G}(S)\right|<t \Delta k$, we can compute the set $N_{G}(S) \backslash S$ in time $O(t \Delta k)$, and construct $\mathcal{T}_{t}^{*}$ in time $\tilde{O}\left(\left|\mathcal{T}_{t-1}\right| t^{2} \Delta k\right)=\tilde{O}\left(n t^{2}(e \Delta k)^{t}\right)$. Finally, we remove duplicates in $\mathcal{T}_{t}^{*}$ to get $\mathcal{T}_{t}$ (e.g., by sorting the sets $S \in \mathcal{T}_{t}^{*}$, where each is represented as a string of length $\left.\widetilde{O}(t)\right)$, in time $\widetilde{O}\left(n t^{3}(e \Delta k)^{t}\right)$. 
Starting from $\mathcal{T}_{1}$, inductively we perform $t$ iterations to construct $\mathcal{T}_{t}$. Thus the overall running time is $\sum_{i=1}^{t} \widetilde{O}\left(n i^{3}(e \Delta k)^{i}\right)=\widetilde{O}\left(n t^{3}(e \Delta k)^{t+1}\right)$.

3.4. Proof of Theorem 3.1. The results in the previous subsection allow us to efficiently enumerate connected sub-insects. To prove Theorem 3.1, it remains to reduce the sum over all (possibly disconnected) $H$ in eq. (10) to a sum over connected $H$. We now show that the method of doing so using Newton's identities and the multiplicativity of the partition function developed by Patel and Regts [41] for graphs extends to the case of insects. Let $G$ be any insect of size $n$ and consider the $t$-th power sum of the inverses of the roots $r_{i}, 1 \leq i \leq n$, of $Z_{G}(\lambda)$ (extended to insects $G$ as in the paragraph following eq. (10)):

$$
p_{t}(G)=\sum_{i=1}^{n} \frac{1}{r_{i}^{t}} \text {. }
$$

Now by Newton's identities (which relate power sums to elementary symmetric polynomials), we have

$$
p_{t}=\sum_{i=1}^{t-1}(-1)^{i-1} p_{t-i} e_{i}+(-1)^{t-1} t e_{t} .
$$

Recall from eq. (10) that $e_{i}$ is a weighted sub-insect count supported on insects of size $\leq i$, and also from Corollary 3.6 that the product of two weighted sub-insect counts supported on insects of size $\leq t_{i}, t_{2}$ respectively is a weighted sub-insect count supported on insects of size $\leq t_{1}+t_{2}$. Therefore, by eq. (11) and induction, each $p_{t}$ is also a weighted sub-insect count supported on insects of size $\leq t$. Thus, for any insect $G$, we may write

$$
p_{t}(G)=\sum_{H \in \mathcal{G}_{t}^{\Delta, k}} a_{H}^{(t)} \cdot \mathbf{1}[H \hookrightarrow G]
$$

for some coefficients $a_{H}^{(t)}$ to be determined. (The superscript $(t)$ reflects the fact that a given $H$ will in general have different coefficients for different $p_{t}$.)

Recall now that if $G$ is disconnected with $G=G_{1} \uplus G_{2}$ then $Z_{G}(\lambda)=Z_{G_{1}}(\lambda) \cdot Z_{G_{2}}(\lambda)$. Thus, the polynomials $Z_{G}(\lambda)$ are multiplicative over $G$, and hence sums of powers of their roots, such as $p_{t}(G)$ are additive: $p_{t}\left(G_{1} \uplus G_{2}\right)=p_{t}\left(G_{1}\right)+p_{t}\left(G_{2}\right)$. Hence by Lemma 3.3, the coefficients of $p_{t}$ are supported on connected insects, and we may write eq. (12) as

$$
p_{t}(G)=\sum_{H \in \mathcal{C}_{t}^{\Delta, k}} a_{H}^{(t)} \cdot \mathbf{1}[H \hookrightarrow G] .
$$

Notice that by Corollary 3.8, there are at most $n(e \Delta k)^{t}$ non-zero terms in this sum.

Lemma 3.10. There is a poly $(n / \varepsilon)$-time algorithm to compute all the coefficients $a_{H}^{(t)}$ in eq. (13), fort $\leq$ $O(\log (n / \varepsilon))$.

Proof. By Lemma 3.9, we compute $\mathcal{T}_{t}$, consisting of all $S \subseteq V(G)$ such that $|S| \leq t$ and $G^{+}[S]$ is connected. As we have removed duplicates, this is exactly $\mathcal{C}_{t}^{\Delta, k}$. We then use dynamic programming to compute the coefficients $a_{H}^{(t)}$.

By eq. (11), for $t=1$ we have $p_{1}=e_{1}$, so by eq. (10) we can read off the coefficients $a_{H}^{(1)}$ from $e_{1}(G)$. Next suppose we have computed $a_{H^{\prime}}^{\left(t^{\prime}\right)}$ for $\left|H^{\prime}\right| \leq t^{\prime}<t$, and we want to compute $a_{H}^{(t)}$ for some fixed connected $H \in \mathcal{C}_{t}^{\Delta, k}$ such that $\mathbf{1}[H \hookrightarrow G]$. Again by eq. (11), it suffices to compute the coefficient corresponding to $H$ in $p_{t-i} e_{i}$ for each $1 \leq i \leq k-1$ (since the contribution of the last term in eq. (11) is 
simply $(-1)^{t-1} t \mu_{H}$ if $|H|=t$ and 0 otherwise). By eqs. (9) and (13), this coefficient is given by

$$
\sum_{\substack{H_{1} \in \mathcal{G}_{i}^{\Delta, k}, H_{2} \in \mathcal{C}_{(t-i)}^{\Delta, k} \\
H_{1} \begin{array}{c}
\text { compatible with } H_{2} \\
H_{1} \cup H_{2}=H
\end{array}}} a_{H_{2}}^{(t-i)} \mu_{H_{1}}^{(i)}=\sum_{\substack{\left(S_{1}, S_{2}\right) \\
S_{1} \cup S_{2}=\underline{V}(H)}} a_{H^{+}\left[S_{2}\right]}^{(t-i)} \mu_{H^{+}\left[S_{1}\right]} .
$$

Since $t \leq O(\log (n / \varepsilon))$, the second sum involves at most $4^{t}=\operatorname{poly}(n / \varepsilon)$ terms. Moreover, due to Corollary 3.8, there are at most $n t(e \Delta k)^{t}=\operatorname{poly}(n / \varepsilon)$ previously computed $a_{H^{\prime}}^{\left(t^{\prime}\right)}$, where $H^{\prime}$ is a connected sub-insect of $G$ and $\left|H^{\prime}\right| \leq t^{\prime}<t$. In order to look up $a_{H^{+}[S]}^{(t-i)}$, one can do a linear scan, which also takes time poly $(n / \varepsilon)$ for $t \leq O(\log (n / \varepsilon))$. The coefficients $\mu_{H^{+}[S]}$ can simply be read off from their definition in eq. (10).

To conclude, because $t \leq O(\log (n / \varepsilon))$, eq. (13) only contains poly $(n / \varepsilon)$ terms. And for each term, $a_{H}^{(t)}$ can be computed using the above dynamic programming scheme in poly $(n / \varepsilon)$ time.

Finally, now that we can compute $a_{H, t}$ efficiently, by eq. (13) we can compute $p_{k}$ using the sub-insect enumerator in Lemma 3.9, and we can then compute $e_{k}$ using Newton's identities as in eq. (11), which completes the proof of Theorem 3.1.

3.5. Proofs of main theorems. Our first main result in the introduction, the FPTAS for the Ising model on graphs throughout the ferromagnetic regime with non-zero field stated in Theorem 1.1, now follows by combining Theorem 3.1 with Lemma 2.1 and the Lee-Yang theorem [29] (also stated as Theorem 4.2 in the next section). Recall from the introduction that the Lee-Yang theorem ensures that the partition function has no zeros inside the unit disk.

Similarly, Theorem 1.4, the FPTAS for two-spin systems on hypergraphs, follows by combining Theorem 3.1 with Lemma 2.1 and the Suzuki-Fisher theorem [53] (also stated as Theorem 4.3 in the next section). Again, the Suzuki-Fisher theorem ensures that there are no zeros inside the unit disk, under the condition on the hyperedge activities stated in Theorem 1.4.

To establish our final main algorithmic result, Theorem 1.3, we first need to prove a new Lee-Yang theorem for the hypergraph Ising model as stated in Theorem 1.2 in the introduction. This will be the content of the next and final section of our paper. Once we have that, Theorem 1.3 follows immediately by the same route as above.

\section{A LeE-YANg Theorem For HyPergraphS}

In this section we prove a tight Lee-Yang theorem for the hypergraph Ising model (Theorem 1.2 in the introduction). We start by extending the definition of the hypergraph Ising model to the multivariate setting, where each vertex and each hyperedge is allowed to have a different activity. As before, we have an underlying hypergraph $G=(V, E)$ with $|V|=n$ vertices. Given vertex activities $\lambda_{1}, \lambda_{2}, \ldots, \lambda_{n}$ and hyperedge activities $\boldsymbol{\beta}=\left(\beta_{e}\right)$, we define

$$
Z_{G}^{\boldsymbol{\beta}}\left(\lambda_{1}, \cdots, \lambda_{n}\right)=\sum_{S \subseteq V} \prod_{e \in E(S, \bar{S})} \beta_{e} \prod_{i \in S} \lambda_{i},
$$

where for a subset $S \subseteq V, E(S, \bar{S})$ is the set of hyperedges with at least one vertex in each of $S$ and $\bar{S}$. Note that

$$
Z_{G}^{\boldsymbol{\beta}}\left(\lambda_{1}, \cdots, \lambda_{n}\right)=\prod_{i=1}^{n} \lambda_{i} \cdot Z_{G}^{\boldsymbol{\beta}}\left(\frac{1}{\lambda_{1}}, \cdots, \frac{1}{\lambda_{n}}\right) .
$$

We use the following definition of the Lee-Yang property. This definition is based on the results of Asano [3] and Suzuki and Fisher [53], and somewhat stricter than the definition used by Ruelle [43]. 
Definition 4.1 (Lee-Yang property). Let $P\left(z_{1}, z_{2}, \ldots, z_{n}\right)$ be a multilinear polynomial. $P$ is said to have the Lee-Yang property (sometimes written as " $P$ is LY") if for any complex numbers $\lambda_{1}, \cdots, \lambda_{n}$ such that $\left|\lambda_{1}\right| \geq 1, \cdots,\left|\lambda_{n}\right| \geq 1$, and $\left|\lambda_{i}\right|>1$ for some $i$, it holds that $P\left(\lambda_{1}, \cdots, \lambda_{n}\right) \neq 0$.

Then the seminal Lee-Yang theorem [29] can be stated as follows:

Theorem 4.2. Let $G$ be a connected undirected graph, and suppose $0<\beta<1$. Then the Ising partition function $Z_{G}^{\beta}\left(\lambda_{1}, \cdots, \lambda_{n}\right)$ has the Lee-Yang property.

The following extension of the Lee-Yang theorem to general symmetric two-spin systems on hypergraphs is due to Suzuki and Fisher [53]. Again the theorem is stated in the multivariate setting, where in the two-spin partition function in eq. (7) each vertex $i$ has a distinct activity $\lambda_{i}$.

Theorem 4.3. Consider any symmetric hypergraph two-spin system, with a connected hypergraph $G$ and edge activities $\left\{\varphi_{e}\right\}$. Then the partition function $Z_{G}^{\varphi}\left(\lambda_{1}, \cdots, \lambda_{n}\right)$ has the Lee-Yang property if $\left|\varphi_{e}(+, \cdots,+)\right| \geq$ $\frac{1}{4} \sum_{\boldsymbol{\sigma} \in\{+,-\}^{V}}\left|\varphi_{e}(\boldsymbol{\sigma})\right|$ for every hyperedge e.

Theorem 4.3 is not tight for the important special case of the Ising model on hypergraphs. Our goal in this section is to prove a tight analog of the original Lee-Yang theorem for this case. Specifically, we will prove the following:

Theorem 4.4. Let $G=(V, E)$ be a connected hypergraph, and $\boldsymbol{\beta}=\left(\beta_{e}\right)_{e \in E}$ be a vector of real valued hyperedge activities so that the activity of edge e $\in E$ is $\beta_{e}$. Then $Z_{G}^{\beta}$ has the Lee-Yang property if the following condition holds for every hyperedge e, where $k \geq 2$ is the size of $e$ :

- if $k=2$, then $-1<\beta_{e}<1$;

- if $k \geq 3$, then $-\frac{1}{2^{k-1}-1}<\beta_{e}<\frac{1}{2^{k-1} \cos ^{k-1}\left(\frac{\pi}{k-1}\right)+1}$.

Further, if the above condition is not satisfied for a given real edge activity $\beta$ and integer $k \geq 2$, then there exists a $k$-uniform hypergraph $H$ with edge activity $\beta$ such that $Z_{H}^{\beta}$ does not have the Lee-Yang property.

Note that the case $k=2$ is just the original Lee-Yang theorem (Theorem 4.2).

The following corollary for the univariate polynomial $Z_{G}^{\boldsymbol{\beta}}(\lambda)$ follows immediately via eq. (15) and the fact that, by Hurwitz's theorem, the zeros of $Z_{G}^{\boldsymbol{\beta}}(\lambda)$ are continuous functions of $\boldsymbol{\beta}$ and thus remain on the unit circle after taking the limit in the range of each $\beta_{e}$.

Corollary 4.5. Let $G=(V, E)$ be a connected hypergraph, and $\boldsymbol{\beta}=\left(\beta_{e}\right)_{e \in E}$ be the vector of real valued hyperedge activities so that the activity of edge $e \in E$ is $\beta_{e}$. Then, all complex zeros of the univariate partition function $Z_{G}^{\boldsymbol{\beta}}(\lambda)$ lie on the unit circle if the following condition holds for every hyperedge e, where $k \geq 2$ is the size of $e$ :

- if $k=2$, then $-1 \leq \beta_{e} \leq 1$;

- if $k \geq 3$, then $-\frac{1}{2^{k-1}-1} \leq \beta_{e} \leq \frac{1}{2^{k-1} \cos ^{k-1}\left(\frac{\pi}{k-1}\right)+1}$.

The corollary establishes the first part of Theorem 1.2 in the introduction, and hence also Theorem 1.3 as explained at the end of the previous section. The second part of Theorem 1.2, which asserts that the range of edge activities under which the theorem holds is optimal, is proven in Section 4.1. (Note that the optimality for the univariate case claimed in Theorem 1.2 does not directly follow from the optimality for the multivariate case guaranteed by Theorem 4.4 above.)

Remark. As a comparison, the result of Suzuki and Fisher, which we restated in Theorem 4.3, implies that a sufficient condition for the Lee-Yang property of $Z_{G}^{\boldsymbol{\beta}}(\lambda)$ is

$$
-\frac{1}{2^{k-1}-1} \leq \beta_{e} \leq \frac{1}{2^{k-1}-1} \text {. }
$$


Note that while the lower bound on $\beta_{e}$ is the same as ours, our (tight) upper bound is always better, and significantly so for the more interesting case of small $k$. For example, for $k=3$ our result gives the optimal range $-\frac{1}{3} \leq \beta_{e} \leq 1$, while the Suzuki-Fisher theorem gives $-\frac{1}{3} \leq \beta_{e} \leq \frac{1}{3}$. Similarly, for $k=4$ the respective ranges are $[-1 / 7,1 / 2]$ (for ours) and $[-1 / 7,1 / 7]$ (for Suzuki-Fisher).

We turn now to the proof of Theorem 4.4. The main technical step in our proof is to derive conditions under which the Ising partition function of a hypergraph consisting of a single hyperedge has the Lee-Yang property. This "base case" turns out to be more difficult than in the case of the original Lee-Yang theorem for graphs. However, as in the graph case, it will turn out that the base case still determines the range of $\beta$ in which the theorem can be claimed to be valid; we show this latter claim, which implies the second part of Theorem 4.4 in Section 4.1.

We begin with the following two lemmas which, taken together, give a partial characterization of the Lee-Yang property. While similar in spirit to the results of Ruelle [43], these lemmas do not follow directly from those results since, as noted above, the version of the Lee-Yang property used here imposes a stricter condition on the polynomial than does the definition used in [43].

Lemma 4.6. Given a multilinear polynomial $P\left(z_{1}, z_{2}, \ldots, z_{n}\right)$ with real coefficients define, for each $1 \leq j \leq$ $n$, multilinear polynomials $A_{j}$ and $B_{j}$ in the variables $z_{1}, \ldots, z_{j-1}, z_{j+1}, \ldots, z_{n}$ such that

$$
P=A_{j} z_{j}+B_{j} .
$$

If $P$ has the Lee-Yang property then, for every $j$ such that the variable $z_{j}$ has positive degree in $P$, it holds that $A_{j}\left(z_{1}, \ldots, z_{j-1}, z_{j+1}, \ldots, z_{n}\right) \neq 0$ when $\left|z_{i}\right| \geq 1$ for all $i \neq j$. In particular, $A_{j}$ itself is $L Y$.

Proof. Without loss of generality, we assume that $j=1$. Note that since $z_{1}$ has positive degree in $P, A_{1}$ is a non-zero polynomial. Suppose that, in contradiction to the claim of the lemma, there exist complex numbers $\lambda_{2}, \ldots, \lambda_{n}$ satisfying $\left|\lambda_{i}\right| \geq 1$ such that $A_{1}\left(\lambda_{2}, \ldots, \lambda_{n}\right)=0$. Since $P$ is LY, it follows that $B_{1}\left(\lambda_{2}, \ldots, \lambda_{n}\right) \neq 0$ (for otherwise, we get a contradiction to the Lee-Yang property by choosing $z_{1}$ to be an arbitrary value outside the closed unit disk).

By continuity, this implies that $\left|B_{1}\right|$ is positive in any small enough neighborhood of $\left(\lambda_{2}, \ldots, \lambda_{n}\right)$ in $\mathbb{C}^{n-1}$. In particular, let $S_{\varepsilon}$ be the open set

$$
S_{\varepsilon}:=\left\{\left(y_{2}, \ldots, y_{n}\right)|| y_{i}-\lambda_{i} \mid<\varepsilon \text { and }\left|y_{i}\right|>1 \text { for } 2 \leq i \leq n\right\} .
$$

Then there exist positive $\delta_{0}$ and $\varepsilon_{0}$ such that $\left|B_{1}\right|$ is at least $\delta_{0}$ in the open set $S_{\varepsilon}$ when $\varepsilon<\varepsilon_{0}$.

Now, since $A_{1}$ is a non-zero multilinear polynomial, it cannot vanish identically on any open set. In particular, it cannot vanish identically in $S_{\varepsilon}$ for any $\varepsilon>0$. On the other hand, since $A_{1}$ vanishes at $\left(\lambda_{2}, \ldots, \lambda_{n}\right)$ it follows from continuity that for $\varepsilon<\varepsilon_{0}$ small enough, $\left|A_{1}\right| \leq \delta_{0} / 2$ in $S_{\varepsilon}$. Since $A_{1}$ does not vanish identically on $S_{\varepsilon}$, there must exist a point $\left(y_{2}, \ldots, y_{n}\right)$ in $S_{\varepsilon}$ such that $0<\left|A_{1}\left(y_{2}, \ldots, y_{n}\right)\right|<\delta_{0} / 2$. Since $\left|B_{1}\left(y_{2}, \ldots, y_{n}\right)\right| \geq \delta_{0}$ by the choice of $\varepsilon_{0}$, it follows that if we define $y_{1}=-B_{1}\left(y_{2}, \ldots, y_{n}\right) / A_{1}\left(y_{2}, \ldots, y_{n}\right)$ then $2<\left|y_{1}\right|<\infty$. However, we then have $P\left(y_{1}, y_{2}, \ldots, y_{n}\right)=0$ even though $\left|y_{1}\right|>1$ and $\left|y_{i}\right| \geq 1$ for all $i$. This contradicts the Lee-Yang property of $P$.

By iterating the above lemma, we get the following corollary.

Corollary 4.7. Let $P\left(z_{1}, z_{2}, \ldots, z_{n}\right)$ be a multilinear polynomial with non-zero real coefficients, i.e.,

$$
P\left(z_{1}, \ldots, z_{n}\right)=\sum_{S \subseteq[n]} p_{S} \prod_{i \in S} z_{i}
$$

where $p_{S} \in \mathbb{R}$ are non-zero for all $S \subseteq[n]$, and assume that $P$ is $L Y$. Then, for every subset $S$ of $[n]$, the polynomial $A_{S}$ defined by the equation

$$
P\left(z_{1}, \ldots, z_{n}\right)=A_{S}\left(\left(z_{i}\right)_{i \notin S}\right) \prod_{i \in S} z_{i}+\sum_{T: S \nsubseteq T} p_{T} \prod_{i \in T} z_{i}
$$

has the property that $A_{S}\left(\left(z_{i}\right)_{i \notin S}\right) \neq 0$ when $\left|z_{i}\right| \geq 1$ for all $i \notin S$. In particular, $A_{S}$ is $L Y$. 
We next show that Lemma 4.6 has a partial converse for symmetric multilinear functions.

Lemma 4.8. Let $P\left(z_{1}, z_{2}, \ldots, z_{n}\right)$ be a symmetric multilinear polynomial with non-zero real coefficients, i.e.,

$$
P\left(z_{1}, \ldots, z_{n}\right)=\sum_{S \subseteq[n]} p_{S} \prod_{i \in S} z_{i}
$$

where $p_{S} \neq 0$ for all $S \subseteq[n]$ and $p_{S}=p_{\bar{S}}$. Assume further that the polynomials $A_{j}$ as defined in Lemma 4.6 all have the property that they are non-zero when all their arguments $z_{i}$ satisfy $\left|z_{i}\right| \geq 1$. Then $P$ is $L Y$.

Proof. We first show that, under our assumptions, if all but one of the $z_{j}$ lie on the unit circle, then $P$ can only vanish if the remaining $z_{j}$ is also on the unit circle. Without loss of generality we set $j=1$, that is, we will show that if $\left|z_{i}\right|=1$ for $i \geq 2$, then any root $z_{1}=\zeta_{1}$ of the equation $A_{1} z_{1}+B_{1}=0$ satisfies $\left|\zeta_{1}\right|=1$. (Here $A_{1}$ and $B_{1}$ are in the notation of Lemma 4.6.)

Since by assumption $A_{1}=\sum_{S \subseteq[2, n]} p_{S \cup\{1\}} \prod_{i \in S} z_{i}$ does not vanish with this setting of the $z_{i}$, we have

$$
\begin{aligned}
\left|\zeta_{1}\right|=\left|\frac{B_{1}}{A_{1}}\right| & =\left|\frac{\sum_{S \subseteq[2, n]} p_{S} \prod_{i \in S} z_{i}}{\sum_{S \subseteq[2, n]} p_{S \cup\{1\}} \prod_{i \in S} z_{i}}\right|=\left|\left(\prod_{i \in[2, n]} z_{i}\right) \frac{\sum_{S \subseteq[2, n]} p_{S} \prod_{\substack{i \notin S \\
i \neq 1}}\left(1 / z_{i}\right)}{\sum_{S \subseteq[2, n]} p_{S \cup\{1\}} \prod_{i \in S} z_{i}}\right| \\
& \stackrel{(\star)}{=}\left|\frac{\sum_{S \subseteq[2, n]} p_{S} \prod_{\substack{i \notin S \\
i \neq 1}} \overline{z_{i}}}{\sum_{S \subseteq[2, n]} p_{S \cup\{1\}} \prod_{i \in S} z_{i}}\right| \stackrel{(\dagger)}{=}\left|\frac{\sum_{S \subseteq[2, n]} p_{S \cup\{1\}} \prod_{i \in S} \overline{z_{i}}}{\sum_{S \subseteq[2, n]} p_{S \cup\{1\}} \prod_{i \in S} z_{i}}\right|=1 .
\end{aligned}
$$

Here $(\star)$ uses the fact that $\left|z_{i}\right|=1$ for $i \geq 2$ and $(\dagger)$ uses the symmetry of $P$. We have thus shown that if $\left(z_{1}, z_{2}, \ldots, z_{n}\right)$ is a zero of $P$ such that $\left|z_{i}\right| \geq 1$ for all $i$ then it is impossible for only one $z_{i}$ to lie outside the closed unit disk.

We now show that if there are $k \geq 2$ values of $i$ for which $z_{i}$ lies outside the closed unit disk, then we can find another zero $\left(\zeta_{1}, \zeta_{2}, \zeta_{3}, \ldots, \zeta_{n}\right)$ of $P$ such that $\left|\zeta_{i}\right| \geq 1$ for all $i$, and exactly $k-1$ of the $\zeta_{i}$ lie outside the closed unit disk. We can then iterate this process to reduce $k$ to 1 , in which case the observation in the previous paragraph leads to a contradiction.

By re-numbering the indices if needed, we can assume that $\left|z_{1}\right|,\left|z_{2}\right|>1$ and $\left|z_{i}\right| \geq 1$ for $i \geq 3$. We can then write

$$
P\left(z_{1}, \ldots, z_{n}\right)=\alpha_{12} z_{1} z_{2}+\alpha_{1} z_{1}+\alpha_{2} z_{2}+\alpha_{\emptyset}
$$

where $\alpha_{12}, \alpha_{1}, \alpha_{2}$ and $\alpha_{\emptyset}$ are non-zero polynomials in $z_{3}, \ldots, z_{n}$. Further, the hypotheses of the lemma imply that $A_{1}=\alpha_{12} z_{2}+\alpha_{1}$ and $A_{2}=\alpha_{12} z_{1}+\alpha_{2}$ both have the Lee-Yang property. Thus, by Lemma 4.6, $\alpha_{12}\left(z_{3}, \ldots, z_{n}\right) \neq 0$ when $\left|z_{i}\right| \geq 1$ for $i \geq 3$. Now, again by hypothesis, $A_{2} \neq 0$ when $\left|z_{1}\right|$ and $\left|z_{3}\right|, \ldots,\left|z_{n}\right|$ are at least 1 , while $z_{1}=-\frac{\alpha_{2}\left(z_{3}, \ldots, z_{n}\right)}{\alpha_{12}\left(z_{3}, \ldots, z_{n}\right)}$ gives $A_{2}=0$. Thus, we must have that

$$
\frac{\left|\alpha_{2}\left(z_{3}, \ldots, z_{n}\right)\right|}{\left|\alpha_{12}\left(z_{3}, \ldots, z_{n}\right)\right|}<1 \text { when }\left|z_{i}\right| \geq 1 \text { for } i \geq 3
$$

We now set $\zeta_{i}=z_{i}$ for $i \geq 3$, and consider $z_{1}$ as a function of $z_{2}$. The equality $P\left(z_{1}, z_{2}, \zeta_{3}, \ldots, \zeta_{n}\right)=0$ is then equivalent to

$$
z_{1}=-\frac{\alpha_{2} z_{2}+\alpha_{\emptyset}}{\alpha_{12} z_{2}+\alpha_{1}}
$$

where the hypotheses of the lemma imply that the denominator (which is equal to $A_{1}\left(z_{2}, \zeta_{3}, \ldots, \zeta_{n}\right)$ ) is non-zero when $\left|z_{2}\right| \geq 1$. We thus see that

$$
\lim _{z_{2} \rightarrow \infty}\left|z_{1}\right|=\frac{\left|\alpha_{2}\right|}{\left|\alpha_{12}\right|}<1
$$

Initially, both $z_{1}$ and $z_{2}$ lie outside the closed unit disk. Thus, by eq. (19) and continuity, we can take $z_{2}$ large enough in absolute value such that $z_{1}$ as defined in eq. (18) lies on the unit circle. We now choose 
$\zeta_{1}$ and $\zeta_{2}$ to be these values of $z_{1}$ and $z_{2}$, respectively, so that we have $P\left(\zeta_{1}, \ldots, \zeta_{n}\right)=0$ and the number of the $\zeta_{i}$ lying on the unit circle is exactly one less than the number of the $z_{i}$ lying on the unit circle, as required.

Along with the above general facts about LY polynomials, we also need the following technical lemma.

Lemma 4.9. Let $m$ be any integer, and $k$ a positive integer such that $2|m| \leq k$. Consider the maximization problem

$$
\begin{aligned}
\max & \prod_{i=1}^{k} \cos \theta_{i} \\
\text { subject to } & -\frac{\pi}{2} \leq \theta_{i} \leq \frac{\pi}{2}, \\
& \sum_{i=1}^{k} \theta_{i}=m \pi
\end{aligned}
$$

The maximum is $\cos ^{k}\left(\frac{m \pi}{k}\right)$, and is attained when $\theta_{i}=\frac{m \pi}{k}$ for all $i$.

Proof. We may assume without loss of generality that $\theta_{i} \in(-\pi / 2, \pi / 2)$ at any maximum (for otherwise the objective value is 0$)$. Now, consider the function $f(x)=\log \cos x$ defined on the interval $(-\pi / 2, \pi / 2)$. Since $f^{\prime}(x)=-\tan x$ is a decreasing function, $f(x)$ is concave for $x \in\left(-\frac{\pi}{2}, \frac{\pi}{2}\right)$. Thus by Jensen's inequality,

$$
\log \prod_{i=1}^{k} \cos \theta_{i}=\sum_{i=1}^{k} f\left(\theta_{i}\right) \leq k f\left(\frac{\sum_{i=1}^{k} \theta_{i}}{k}\right) \leq k \log \cos \left(\frac{m \pi}{k}\right),
$$

and equality holds when $\theta_{i}=\frac{m \pi}{k}$ for all $i$. Note that these $\theta_{i}$ are in $(-\pi / 2, \pi / 2)$ since $2|m| \leq k$.

We are now ready to tackle the case of a single hyperedge.

Lemma 4.10. Fix an integer $k \geq 2$ and a hyperedge activity $\beta \in \mathbb{R}$. Let $G=\left(V=\left\{v_{1}, v_{2}, \ldots, v_{k}\right\}, E=\right.$ $\left.\left\{\left\{v_{1}, v_{2}, \ldots, v_{k}\right\}\right\}\right)$ be a hypergraph consisting of a single hyperedge of size $k$ and activity $\beta$. If $k=2$ and $\beta \in(-1,1)$, or $k \geq 3$ and $\beta$ satisfies

$$
-\frac{1}{2^{k-1}-1}<\beta<\frac{1}{2^{k-1} \cos ^{k-1}\left(\frac{\pi}{k-1}\right)+1},
$$

then the partition function $Z_{G}^{\beta}$ has the Lee-Yang property.

Remark. Note that the condition on $\beta$ imposed above is monotone in $k$ : i.e., if $\beta$ is such that the partition function of a hyperedge of size $k \geq 2$ is LY, then for the same $\beta$ the partition function of a hyperedge of size $k^{\prime}<k$ is also LY.

Proof. For $k=2$, the lemma is a special case of the Lee-Yang theorem [29] (although it also follows by specializing the argument below). We therefore assume $k \geq 3$.

Since the Ising partition function is symmetric and all terms in the polynomial appear with positive coefficients, Lemma 4.8 applies and it suffices to verify that the polynomials $A_{j}$ do not vanish when $\left|z_{i}\right| \geq 1$ for $i \neq j$. Without loss of generality we fix $j=1$. We then have

$$
A_{1}=\beta \prod_{i=2}^{k}\left(1+z_{i}\right)+(1-\beta) \prod_{i=2}^{k} z_{i} .
$$


Thus $A_{1}=0$ is equivalent to

$$
\frac{1}{\beta}=1-\prod_{i=2}^{k}\left(1+\frac{1}{z_{i}}\right) .
$$

To establish the lemma, we therefore only need to show that for the claimed values of $\beta$, eq. (20) has no solutions when $\left|z_{i}\right| \geq 1$ for all $i \geq 2$. We now proceed to establish this by analyzing the product on the right hand side of eq. (20).

The map $z \mapsto 1+1 / z$ is a bijection from the complement of the open unit disk to the closed disk $D$ of radius 1 centered at 1 . Any $y \in D$ can be written as $y=r \exp (\iota \theta)$ for $\theta \in[-\pi / 2, \pi / 2]$ and $0 \leq r \leq 2 \cos \theta$. Consider now the set $\mathbb{R} \cap\left\{\prod_{i=2}^{k} y_{i} \mid y_{i} \in D\right.$ for $\left.2 \leq i \leq k\right\}$. We show that, for $k \geq 3$, this set is exactly the interval $\left[-\tau_{0}, \tau_{1}\right]$ where $\tau_{0}=2^{k-1} \cos ^{k-1}(\pi /(k-1))$ and $\tau_{1}=2^{k-1}$. The claim of the lemma then follows since for the given values of $\beta, 1-1 / \beta$ lies outside $\left[-\tau_{0}, \tau_{1}\right]$ and hence eq. (20) cannot hold.

Recalling that each $y \in D$ can be written in the form $r \exp (\iota \theta)$ where $\theta \in[-\pi / 2, \pi / 2]$ and $0 \leq r \leq$ $2 \cos \theta$, we find that the values $\tau_{0}$ and $\tau_{1}$ are defined by the following optimization problems (both of which are feasible since $k \geq 3$ ):

$$
\begin{aligned}
& \tau_{0}=2^{k-1} \max \prod_{i=2}^{k} \cos \theta_{i} \quad \tau_{1}=2^{k-1} \max \prod_{i=2}^{k} \cos \theta_{i} \\
& \text { subject to }-\frac{\pi}{2} \leq \theta_{i} \leq \frac{\pi}{2}, \quad \text { subject to }-\frac{\pi}{2} \leq \theta_{i} \leq \frac{\pi}{2} \text {, } \\
& \sum_{i=2}^{k} \theta_{i}=(2 n+1) \pi \quad \sum_{i=2}^{k} \theta_{i}=2 n \pi \\
& \text { for some } n \in \mathbb{Z} \\
& \text { s.t. }|2 n+1| \leq(k-1) / 2 . \quad \text { s.t. }|n| \leq(k-1) / 4 \text {. }
\end{aligned}
$$

Using Lemma 4.9, we then see that $\tau_{0}=2^{k-1} \cos ^{k-1}(\pi /(k-1))$ and $\tau_{1}=2^{k-1}$, as required.

We now proceed to an inductive proof of Theorem 4.4, using Lemma 4.10 as the base case.

Proof of Theorem 4.4. The case $k=2$ is a special case of the Lee-Yang theorem [29] (though, as with the proof of Lemma 4.10, the argument below can again be specialized to directly establish this). We assume therefore that $k \geq 3$.

The proof uses the inductive method of Asano [3]. When the hypergraph consists of a single hyperedge of size $k^{\prime} \leq k$, it follows from Lemma 4.10 and the remark immediately after it that the partition function is LY for the claimed values of the edge activity $\beta$. For the induction, we use the fact that the Lee-Yang property of the partition function is preserved under the following two operations:

(1) Adding a hyperedge: In this operation, a new hyperedge $e$ of size $k^{\prime} \leq k$ and activity $\beta_{e}$ as claimed in the statement of the theorem, is added to a connected hypergraph in such a way that exactly one of its $k^{\prime}$ vertices already exists in the starting hypergraph, while the other $k^{\prime}-1$ vertices are new. Note that this operation keeps the hypergraph connected. We assume that the partition functions of both the original hypergraph as well as the newly added edge separately have the Lee-Yang property: this follows from the induction hypothesis (for the hypergraph) and Lemma 4.10 (for the new hyperedge).

(2) Asano contraction: In this operation, two vertices $u^{\prime}, u^{\prime \prime}$ in a connected hypergraph that are not both included in any one hyperedge are merged so that the new merged vertex $u$ is incident on all the hyperedges incident on $u^{\prime}$ or $u^{\prime \prime}$ in the original graph. Note that this operation keeps the hypergraph connected and does not change the size of any of the hyperedges. 
Any connected non-empty hypergraph $G$ can be constructed by starting with any arbitrary hyperedge present in $G$ and performing a finite sequence of the above two operations: to add a new hyperedge $e$ with activity $\beta_{e}$, one first uses operation 1 to add a hyperedge which has the same activity $\beta_{e}$ and has new copies of all but one of the incident vertices of $e$, and then uses operation 2 to merge these new copies with their counterparts, if any, in the previous hypergraph. Note that in this process, a hyperedge $e$ can be added only when at least one of its vertices is already included in the current hypergraph. However, since $G$ is assumed to be connected, its hyperedges can be ordered so that all of them are added by the above process. Thus, assuming that the above two operations preserve the Lee-Yang property, it follows by induction that the partition functions of all connected hypergraphs of hyperedge size at most $k$, and edge activities $\beta_{e}$ as claimed in the theorem, have the Lee-Yang property.

Given Corollary 4.7, it can be proved, by adapting an argument first developed by Asano [3], that these two operations preserve the Lee-Yang property. Asano's method has by now become standard (see, e.g., [53, Propositions 1, 2]), but we include the details here for completeness.

Consider first operation 1 . Let $G$ be the original hypergraph and $H$ the new hyperedge (with $k^{\prime} \leq k$ vertices) being added, and assume, by renumbering vertices if required, that the single shared vertex is $v_{1}$ in $G$ and $u_{1}$ in $H$, respectively. Let $P\left(z_{1}, z_{2}, \ldots, z_{n}\right)=A\left(z_{2}, \ldots, z_{n}\right) z_{1}+B\left(z_{2}, \ldots, z_{n}\right)$ and $Q\left(y_{1}, y_{2}, \ldots, y_{k^{\prime}}\right)=C\left(y_{2}, \ldots, y_{k^{\prime}}\right) y_{1}+D\left(y_{2}, \ldots, y_{k^{\prime}}\right)$ be the Ising partition functions of $G$ and $H$, respectively, where $z_{1}$ and $y_{1}$ are the variables corresponding to $v_{1}$ and $u_{1}$, respectively. Both $P$ and $Q$ are LY by the hypothesis of the operation. The partition function $R$ of the new graph can be written as

$$
R\left(z, z_{2}, \ldots, z_{n}, y_{2}, \ldots, y_{k^{\prime}}\right)=A\left(z_{2}, \ldots, z_{n}\right) C\left(y_{2}, \ldots, y_{k^{\prime}}\right) z+B\left(z_{2}, \ldots, z_{n}\right) D\left(y_{2}, \ldots, y_{k^{\prime}}\right),
$$

where $z$ is a new variable corresponding to the new vertex created by the merger of $u_{1}$ and $v_{1}$. Let $\lambda_{2}, \ldots, \lambda_{n}, \mu_{2}, \ldots, \mu_{k^{\prime}}$ be complex numbers lying outside the open unit disk. In order to prove that $R$ is LY, we need to show that (i) $R\left(z, \lambda_{2}, \ldots, \lambda_{n}, \mu_{2}, \ldots, \mu_{k^{\prime}}\right)=0$ implies that $|z| \leq 1$; and (ii) when at least one of these complex numbers lies strictly outside the closed unit disk then $R\left(z, \lambda_{2}, \ldots, \lambda_{n}, \mu_{2}, \ldots, \mu_{k^{\prime}}\right)=0$ implies that $|z|<1$. Now, since $P$ and $Q$ are assumed to be LY, Lemma 4.6 implies that $A=A\left(\lambda_{2}, \ldots, \lambda_{n}\right)$ and $C=C\left(\mu_{2}, \ldots, \mu_{k^{\prime}}\right)$ are both non-zero. Thus, $R=0$ implies that

$$
|z|=|B / A| \cdot|D / C|,
$$

where $B=B\left(\lambda_{2}, \ldots \lambda_{n}\right)$ and $D=D\left(\mu_{2}, \ldots, \mu_{k^{\prime}}\right)$. Since all the $\lambda_{i}$ and $\mu_{i}$ lie outside the open unit disk and $P$ and $Q$ are LY, $|B / A|,|D / C| \leq 1$, so that from eq. (21), $|z| \leq 1$. This establishes condition (i). Further, when at least one of the $\lambda_{i}$ lies strictly outside the closed unit disk, then again, since $P$ is LY, $|B / A|<1$. Similarly, $|D / C|<1$ when one of the $\mu_{i}$ lies outside the closed unit disk. Thus, when at least one of the $\lambda_{i}$ and the $\mu_{i}$ lies outside the closed unit disk, it follows from eq. (21) that $|z|<1$, thus establishing condition (ii) and concluding the argument that $R$ is LY.

We now consider operation 2. By renumbering vertices if necessary, let $v_{1}$ and $v_{2}$ be the vertices to be merged. The partition function $P$ of the original graph (where $v_{1}$ and $v_{2}$ are not merged) can be written as

$$
P\left(z_{1}, z_{2}, z_{3}, \ldots, z_{n}\right)=A\left(z_{3}, \ldots, z_{n}\right) z_{1} z_{2}+B\left(z_{3}, \ldots, z_{n}\right) z_{1}+C\left(z_{3}, \ldots, z_{n}\right) z_{2}+D,
$$

and is LY by the hypothesis of the operation. The partition function $R$ after the merger is then given by

$$
R\left(z, z_{3}, \ldots, z_{n}\right)=A\left(z_{3}, \ldots, z_{n}\right) z+D
$$

where $z$ is a new variable corresponding to the vertex created by the merger of $v_{1}$ and $v_{2}$. Now, let $\lambda_{3}, \ldots, \lambda_{n}$ be complex numbers lying outside the open unit disk. Corollary 4.7 implies that $A=A\left(\lambda_{3}, \ldots, \lambda_{n}\right) \neq 0$. Thus, $R\left(z, \lambda_{3}, \ldots, \lambda_{n}\right)=0$ implies that

$$
|z|=\left|D\left(\lambda_{3}, \ldots, \lambda_{n}\right) / A\left(\lambda_{3}, \ldots, \lambda_{n}\right)\right|=|D / A| .
$$

Now, since $P$ is LY, both zeros of the quadratic equation $P\left(x, x, \lambda_{3}, \ldots, \lambda_{n}\right)=0$ satisfy $|x| \leq 1$, and indeed, $|x|<1$ when at least one of the $\lambda_{i}$ lies strictly outside the closed unit disk. Thus, the product $D / A$ of its zeros also satisfies $|D / A| \leq 1$, and further satisfies the stronger inequality $|D / A|<1$ in case at least 
one of the $\lambda_{i}$ lies strictly outside the closed unit disk. Eq. (22) then implies that $|z| \leq 1$ in the first case and $|z|<1$ in the second case, which establishes that $R$ is LY.

This concludes the proof of the first part of Theorem 4.4. We now prove the optimality of the conditions imposed on the edge parameters. In the case $k=2$, this follows by considering the partition function $z_{1} z_{2}+\beta z_{1}+\beta z_{2}+1$ of a single edge. When $\beta>1$ (respectively, when $\beta<-1$ ), $z_{1}=z_{2}=-\beta-\sqrt{\beta^{2}-1}$ (respectively, $z_{1}=z_{2}=-\beta+\sqrt{\beta^{2}-1}$ ) is a zero of the partition function satisfying $\left|z_{1}\right|,\left|z_{2}\right|>1$ and hence contradicting the Lee-Yang property. Similarly $z_{1}=-1, z_{2}=2$ when $\beta=1$, and $z_{1}=1, z_{2}=2$ when $\beta=-1$, are zeros which contradict the Lee-Yang property.

We now consider the case $k \geq 3$. In this case, we take our example to be the single hyperedge of size $k$ and consider its partition function

$$
P\left(z_{1}, z_{2}, \ldots, z_{k}\right):=\beta \prod_{i=1}^{k}\left(1+z_{i}\right)+(1-\beta)\left(1+\prod_{i=1}^{k} z_{i}\right) .
$$

Our strategy is to show that when

$$
\beta \notin\left(-\frac{1}{2^{-k-1}-1}, \frac{1}{2^{k-1} \cos ^{k-1}\left(\frac{\pi}{k-1}\right)+1}\right),
$$

the polynomial $A_{1}\left(z_{2}, z_{2}, \ldots, z_{k}\right)$, which is the coefficient of $z_{1}$ in $P$ as defined in Lemma 4.6, vanishes at a point with $\left|z_{i}\right| \geq 1$ for $i \geq 2$. It then follows from Lemma 4.6 that $P$ cannot have the Lee-Yang property.

To carry out the strategy, we reuse some of the notation and calculations from the proof of Lemma 4.10. Let $D$ be the closed disk of radius 1 centered at 1, as defined in the proof of that lemma. Eq. (20), taken together with the discussion following it, implies that finding a zero of $A_{1}\left(z_{2}, \ldots, z_{k}\right)$ with $\left|z_{i}\right| \geq 1$, $2 \leq i \leq k$, is equivalent to finding $y_{i} \in D, y_{i} \neq 1$ such that $1-\frac{1}{\beta}=\prod_{i=2}^{k} y_{i}$. We can in fact choose all the $y_{i}$ to be equal, so that using the same representation of $D$ as in the proof of Lemma 4.10, our task reduces to finding an angle $\theta \in[-\pi / 2, \pi / 2]$ and $0 \leq r \leq 2 \cos \theta$ such that $y_{i}=r e^{\iota \theta}, 2 \leq i \leq k$, and

$$
1-\frac{1}{\beta}=\left(r e^{\iota \theta}\right)^{k-1} \text {. }
$$

Let $\gamma:=1-\frac{1}{\beta}$. We now partition the condition on $\beta$ in (24) into three different cases. Suppose first that $\beta \leq-\frac{1}{2^{k-1}-1}$. This is equivalent to $1<\gamma \leq 2^{k-1}$. In this case $\theta=0, r=\gamma^{\frac{1}{k-1}} \in(1,2]$ gives a desired solution to (25) (note that we have $y_{i} \in(1,2]$ in this case). The same solution for $\theta$ and $r$ also works when $\beta>1$ (in which case $0<\gamma<1$ and $y_{i} \in(0,1)$ ). The remaining case is $1 \geq \beta \geq \frac{1}{2^{k-1} \cos ^{k-1}\left(\frac{\pi}{k-1}\right)+1}$, which in turn is equivalent to $-2^{k-1} \cos ^{k-1}\left(\frac{\pi}{k-1}\right) \leq \gamma \leq 0$, and $\theta=\frac{\pi}{k-1}, r=|\gamma|^{\frac{\pi}{k-1}} \leq 2 \cos \theta$ gives a solution in this case.

4.1. Optimality of the univariate hypergraph Lee-Yang theorem. We now prove the second part of the univariate hypergraph Lee-Yang theorem, Theorem 1.2, i.e., that the range of edge activities under which the first part of that theorem holds is optimal. The tight example for the case $k=2$ is again a single edge, and as observed above, the roots of the univariate partition function of the edge when $|\beta|>1$ are $-\beta \pm \sqrt{\beta^{2}-1}$, which do not lie on the unit circle.

We now consider the case $k \geq 3$. The tight example is again a hyperedge of size $k^{\prime} \leq k$. The partition function $P_{k^{\prime}}(z)$ of this graph is

$$
P_{k^{\prime}}(z):=\beta(1+z)^{k^{\prime}}+(1-\beta)\left(1+z^{k^{\prime}}\right),
$$

and we will show that it has at least one root outside the unit circle when $\beta \neq 1$ satisfies

$$
\beta \notin\left[-\frac{1}{2^{-k-1}-1}, \frac{1}{2^{k-1} \cos ^{k-1}\left(\frac{\pi}{k-1}\right)+1}\right] .
$$


We consider three exhaustive cases under which (26) holds.

Case 1: $\beta>1$ : In this case our tight example is a hyperedge of size $k^{\prime}=2 \leq k$, and the result follows from that of the case $k=2$.

Case 2: $\beta<-\frac{1}{2^{k-1}-1}$ : In this case, our example is a hyperedge of size $k$. We note then that $P_{k}(0)=1$ and $P_{k}(1)=2 \beta\left(2^{k-1}-1\right)+2<0$. Thus, there exists a $z$ in the interval $(0,1)$ for which $P_{k}(z)=0$, and hence $P_{k}$ has a zero that is not on the unit circle.

Case 3: $\frac{1}{2^{k-1} \cos ^{k-1}\left(\frac{\pi}{k-1}\right)+1}<\beta<1$ : Our tight example is again a hyperedge of size $k$. We will show that the degree $k$ polynomial $P_{k}$ has at most $k-3$ zeros (counting with multiplicities) on the unit circle $C$, and hence must have at least one zero outside it.

We first consider the point $z=-1$. Note that since $\beta \neq 1, P_{k}(-1)=0$ if and only if $k$ is odd, and in this case $P_{k}^{\prime}(-1)=k(1-\beta) \neq 0$. Therefore, -1 is a zero of multiplicity 1 of $P_{k}$ when $k$ is odd, and is not a zero of $P_{k}$ when $k$ is even.

We now consider zeros of $P_{k}$ in $C \backslash\{-1\}$. Let $\tau:=2^{k-1} \frac{\beta}{\beta-1}$ and $g(z):=\frac{1+z^{k}}{(1+z)^{k}}$. Note that any $z \in C \backslash\{-1\}$ is a zero of multiplicity $l$ of $P_{k}$ if and only if it is a zero of the same multiplicity $l$ of the meromorphic function $g(z)-\tau / 2^{k-1}$. In particular, at such a $z$, the order of the first non-zero derivative of $P_{k}$ is the same as the order of the first non-zero derivative of $g$, and this number is the multiplicity of $z$ as a zero of $P$ (or equivalently, as a root of $g(z)=\tau / 2^{k-1}$ ). Note also that $g(z)$ maps $C \backslash\{-1\}$ into the real line: in fact, for $z=e^{2 \iota \theta}, \theta \in(-\pi / 2, \pi / 2)$, we have

$$
2^{k-1} g(z)=2^{k-1} \cdot \frac{1+\cos 2 k \theta+\iota \sin 2 k \theta}{(1+\cos 2 \theta+\iota \sin 2 \theta)^{k}}=\frac{2^{k} \cos k \theta}{(2 \cos \theta)^{k}} \cdot \frac{e^{\iota k \theta}}{e^{\iota k \theta}}=\frac{\cos k \theta}{\cos ^{k} \theta}=: h(\theta),
$$

and further $h^{\prime}(\theta)=2^{k} \iota z g^{\prime}(z)$, so that $h^{\prime}(\theta)=0$ if and only if $g^{\prime}(z)=0$. Indeed, by computing further derivatives, one sees that the multiplicity of any root of $h(\theta)=\tau$ in $(-\pi / 2, \pi / 2)$ (i.e., the order of the first non-zero derivative of $h$ at the root) is the same as the multiplicity of the corresponding root $z=e^{2 \iota \theta}$ of $g(z)=\tau / 2^{k-1}$.

Thus, in order to establish our claim that $P_{k}(z)$ has at most $k-3$ zeros (counting with multiplicities and also accounting for the possible zero at -1 considered above) on the unit circle $C$, we only need to show that the number of roots of the equation $h(\theta)=\tau$ on $(-\pi / 2, \pi / 2)$ (counted with multiplicities) is at most $k-4$. We now proceed to establish this property of $h$. Note that for the range of $\beta$ being considered, we have $\tau<-\sec ^{k-1}\left(\frac{\pi}{k-1}\right)$.

Since $h(\theta)=h(-\theta)$, we consider its behavior only in the interval $I=[0,-\pi / 2)$. We have $h^{\prime}(\theta)=-\frac{k \sin (k-1) \theta}{\cos ^{k+1} \theta}$, so that the zeros of $h^{\prime}$ in $I$ are given by $\rho_{i}=i \pi /(k-1)$, where $0 \leq i<\lfloor k / 2\rfloor$ is an integer. Note that all these zeros of $h^{\prime}$ are in fact simple: $h^{\prime \prime}\left(\rho_{i}\right) \neq 0$. Thus, any root of $h(\theta)=\tau$ is of multiplicity at most 2 . Now, define $\rho_{\lfloor k / 2\rfloor}=\pi / 2$, and let $I_{i}$ be the interval $\left[\rho_{i}, \rho_{i+1}\right)$ for $0 \leq i \leq\lfloor k / 2\rfloor-1$. We note the following facts (see Figure 1 for an example):

(1) In the interval $I_{i}, h$ is strictly decreasing when $i$ is even and strictly increasing when $i$ is odd.

(2) For $i<\lfloor k / 2\rfloor, h\left(\rho_{i}\right)=(-1)^{i} \sec ^{k-1}\left(\frac{i \pi}{k-1}\right)$, so that $h\left(\rho_{i}\right)$ is strictly positive when $i$ is even and strictly negative when $i$ is odd. Further, $h\left(\rho_{1}\right)=-\sec ^{k-1}\left(\frac{\pi}{k-1}\right)>\tau$.

From these observations we can now deduce that when $-\sec ^{k-1}\left(\frac{\pi}{k-1}\right)>\tau, h(\theta)=\tau$ has

(1) no roots in $I_{0}$ and $I_{1}$,

(2) at most two roots in $I_{i} \cup I_{i+1}$, counting multiplicities, when $i$ is a positive even integer strictly less than $\lfloor k / 2\rfloor-1$. The two roots can arise in only the following two ways: there can be one root each, with multiplicity 1 , in each of the two intervals $I_{i}$ and $I_{i+1}$, or else there can be a root of multiplicity 2 at $\rho_{i+1}$.

(3) at most one additional root in $I_{\lfloor k / 2\rfloor-1}$, and this additional root can arise only when $\lfloor k / 2\rfloor-1$ is even. 


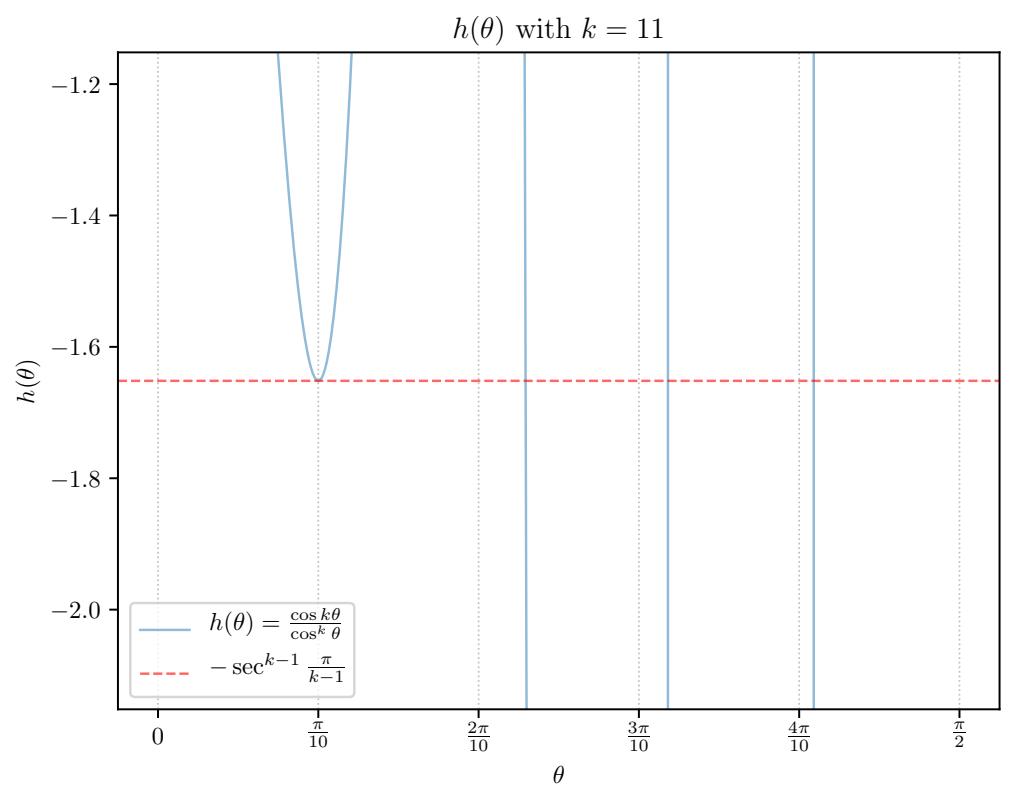

FIGURE 1. The function $h(\theta)$

Together, the above three items imply that when $\tau<-\sec ^{k-1}\left(\frac{\pi}{k-1}\right)$, the number of roots of $h(\theta)=\tau$ in $I=[0,-\pi / 2)$, counted with their multiplicities, is at most $\lfloor k / 2\rfloor-2$. Using the symmetry of $h$ around 0 pointed out above, we thus see that the number of roots of $h(\theta)-\tau$ in $(-\pi / 2, \pi / 2)$ is at most $k-4$, so that $P_{k}$ has at most $k-3$ zeros (accounting for the possible simple zero at -1 when $k$ is odd) on the unit circle for such $\beta$. This implies that at least one zero of the degree $k$ polynomial $P_{k}$ must lie outside the unit circle, as required.

\section{ACKNOWLEDGMENTS}

We thank Alexander Barvinok, Guus Regts and anonymous reviewers for helpful comments. JL and AS are supported in part by US NSF grant CCF-1420934. PS is supported by a Ramanujan Fellowship of the Indian Department of Science and Technology. Some of this work was done at the Simons Institute for the Theory of Computing at UC Berkeley.

\section{REFERENCES}

[1] N. Anari and S. O. Gharan. The Kadison-Singer problem for strongly Rayleigh measures and applications to Asymmetric TSP. In Proc. 56th IEEE Symp. Found. Comp. Sci. (FOCS), 2015.

[2] N. Anari and S. O. Gharan. A generalization of permanent inequalities and applications in counting and optimization. In Proc. 49th ACM Symp. Theory Comput. (STOC), pages 384-396, 2017. arXiv:1702.02937.

[3] T. Asano. Lee-Yang theorem and the Griffiths inequality for the anisotropic Heisenberg ferromagnet. Phys. Rev. Let., 24(25):14091411, 1970.

[4] J. C. A. Barata and P. S. Goldbaum. On the distribution and gap structure of Lee-Yang zeros for the Ising model: Periodic and aperiodic couplings. F. Stat. Phys., 103(5-6):857-891, 2001.

[5] J. C. A. Barata and D. H. U. Marchetti. Griffiths' singularities in diluted Ising models on the Cayley tree. F. Stat. Phys., 88(1-2):231-268, 1997.

[6] A. Barvinok. Computing the partition function for cliques in a graph. Theory Comput., 11(13):339-355, 2015.

[7] A. Barvinok. Computing the permanent of (some) complex matrices. Found. Comput. Math., 16(2):329-342, 2015.

[8] A. Barvinok. Combinatorics and Complexity of Partition Functions. Springer, 2016.

[9] A. Barvinok and P. Soberón. Computing the partition function for graph homomorphisms. Combinatorica, 2016. [Online]. 
[10] A. Barvinok and P. Soberón. Computing the partition function for graph homomorphisms with multiplicities. F. Combin. Theory, Ser. A, 137:1-26, 2016.

[11] N. Berger, C. Kenyon, E. Mossel, and Y. Peres. Glauber dynamics on trees and hyperbolic graphs. Probab. Theory Relat. Fields, 131(3):311-340, 2005.

[12] J. Borcea and P. Brändén. The Lee-Yang and Pólya-Schur programs. I. Linear operators preserving stability. Invent. Math., 177(3):541-569, 2009.

[13] J. Borcea and P. Brändén. The Lee-Yang and Pólya-Schur programs. II. Theory of stable polynomials and applications. Comm. Pure Appl. Math., 62(12):1595-1631, 2009.

[14] J. Borcea, P. Brändén, and T. Liggett. Negative dependence and the geometry of polynomials. f. AMS, 22(2):521-567, 2009.

[15] C. Borgs, J. Chayes, J. Kahn, and L. Lovász. Left and Right Convergence of Graphs with Bounded Degree. Random Struct. Algorithms, 42(1):1-28, 2013.

[16] J.-Y. Cai, X. Chen, and P. Lu. Graph homomorphisms with complex values: A dichotomy theorem. In Proc. ICALP, volume 6198 of Lecture Notes in Computer Science, pages 275-286. Springer, 2010.

[17] P. Csikvári and P. E. Frenkel. Benjamini-Schramm continuity of root moments of graph polynomials. Eur. F. Combin., 52:302-320, 2016.

[18] C. Efthymiou, T. P. Hayes, D. Štefankovic, E. Vigoda, and Y. Yin. Convergence of MCMC and loopy BP in the tree uniqueness region for the hard-core model. In Proc. 57th IEEE Symp. Found. Comp. Sci. (FOCS), pages 704-713, 2016.

[19] A. Galanis, Q. Ge, D. Štefankovič, E. Vigoda, and L. Yang. Improved inapproximability results for counting independent sets in the hard-core model. Random Struct. Algorithms, 45(1):78-110, 2014.

[20] A. Galanis and L. A. Goldberg. The complexity of approximately counting in 2-spin systems on $k$-uniform bounded-degree hypergraphs. Inf. Comput., 251:36-66, 2016.

[21] L. A. Goldberg, M. Grohe, M. Jerrum, and M. Thurley. A complexity dichotomy for partition functions with mixed signs. SIAM f. Comput., 39(7):3336-3402, 2010.

[22] L. A. Goldberg, M. Jerrum, and M. Paterson. The computational complexity of two-state spin systems. Random Struct. Algorithms, 23(2):133-154, 2003.

[23] H. Guo and M. Jerrum. Random cluster dynamics for the Ising model is rapidly mixing. In Proc. 28th ACM-SIAM Symp. Discrete Algorithms (SODA), pages 1818-1827, 2017.

[24] H. Guo and P. Lu. Uniqueness, spatial mixing, and approximation for ferromagnetic 2-spin systems. In Proc. APPROX/RANDOM, 2016.

[25] E. Ising. Beitrag zur Theorie des Ferromagnetismus. Z. Phys., 31:253-258, 1925.

[26] M. Jerrum and A. Sinclair. Approximating the permanent. SIAM F. Comput., 18(6):1149-1178, 1989.

[27] M. Jerrum and A. Sinclair. Polynomial-time approximation algorithms for the Ising model. SIAM f. Comput., 22(5):1087-1116, 1993.

[28] M. Jerrum, L. G. Valiant, and V. V. Vazirani. Random generation of combinatorial structures from a uniform distribution. Theor. Comput. Sci., 43:169-188, 1986.

[29] T. D. Lee and C. N. Yang. Statistical theory of equations of state and phase transitions. II. Lattice gas and Ising model. Phys. Rev., 87(3):410-419, 1952.

[30] L. Li, P. Lu, and Y. Yin. Correlation decay up to uniqueness in spin systems. In Proc. 24th ACM-SIAM Symp. Discrete Algorithms (SODA), pages 67-84, 2013.

[31] Y. Long, A. Nachmias, W. Ning, and Y. Peres. A power law of order $1 / 4$ for critical mean-field Swendsen-Wang dynamics. Memo. AMS, 232(1092), 2014.

[32] P. Lu, K. Yang, and C. Zhang. FPTAS for hardcore and Ising models on hypergraphs. In Proc. 33rd Symp. Theoret. Aspects Comp. Sci. (STACS), pages 51:1-51:14, 2016.

[33] E. Lubetzky and A. Sly. Critical Ising on the square lattice mixes in polynomial time. Comm. Math. Phys., 313(3):815-836, 2012.

[34] M. Luby and E. Vigoda. Approximately counting up to four. In Proc. 29th ACM Symp. Theory. Comput., pages 682-687, 1997.

[35] A. Marcus, D. Spielman, and N. Srivastava. Interlacing families I: Bipartite Ramanujan graphs of all degrees. Ann. Math., pages 307-325, 2015.

[36] A. W. Marcus, D. A. Spielman, and N. Srivastava. Interlacing families II: Mixed characteristic polynomials and the KadisonSinger problem. Ann. Math., 182:327-350, 2015.

[37] F. Martinelli and E. Olivieri. Approach to equilibrium of Glauber dynamics in the one phase region. I. The attractive case. Comm. Math. Phys., 161(3):447-486, 1994.

[38] F. Martinelli and E. Olivieri. Approach to equilibrium of Glauber dynamics in the one phase region: II. The general case. Comm. Math. Phys., 161:487-514, 1994.

[39] F. Martinelli, A. Sinclair, and D. Weitz. Glauber dynamics on trees: Boundary conditions and mixing time. Comm. Math. Phys., 250(2):301-334, 2004.

[40] E. Mossel and A. Sly. Exact thresholds for Ising-Gibbs samplers on general graphs. Ann. Probab., 41(1):294-328, 2013.

[41] V. Patel and G. Regts. Deterministic polynomial-time approximation algorithms for partition functions and graph polynomials. SIAM J. Comput., 46(6):1893-1919, Dec. 2017. arXiv:1607.01167. 
[42] D. Randall and D. Wilson. Sampling spin configurations of an Ising system. In Proc. 10th ACM-SIAM Symp. Discrete Algorithms (SODA), pages 959-960, 1999.

[43] D. Ruelle. Characterization of Lee-Yang polynomials. Ann. Math., 171(1):589-603, 2010.

[44] A. Scott and A. Sokal. The Repulsive Lattice Gas, the Independent-Set Polynomial, and the Lovász Local Lemma. F. Stat. Phys., 118(5-6):1151-1261, 2004.

[45] J. B. Shearer. On a problem of Spencer. Combinatorica, 5(3), 1985.

[46] A. Sinclair and P. Srivastava. Lee-Yang theorems and the complexity of computing averages. Comm. Math. Phys., 329(3):827-858, 2014.

[47] A. Sinclair, P. Srivastava, and M. Thurley. Approximation algorithms for two-state anti-ferromagnetic spin systems on bounded degree graphs. F. Stat. Phys., 155(4):666-686, 2014.

[48] A. Sinclair, P. Srivastava, D. Štefankovič, and Y. Yin. Spatial mixing and the connective constant: Optimal bounds. Probab. Theory Relat. Fields, pages 1-45, 2016.

[49] A. Sly and N. Sun. Counting in two-spin models on $d$-regular graphs. Ann. Probab., 42(6):2383-2416, 2014.

[50] R. Song, Y. Yin, and J. Zhao. Counting hypergraph matchings up to uniqueness threshold. In Proc. APPROX/RANDOM, pages 46:1-46:29, 2016.

[51] R. Stanley and S. Fomin. Enumerative Combinatorics. Cambridge University Press, 1999.

[52] D. Straszak and N. K. Vishnoi. Real stable polynomials and matroids: Optimization and counting. In Proc. 49th ACM Symp. Theory Comput. (STOC), pages 370-383, 2017. arXiv:1611.04548.

[53] M. Suzuki and M. E. Fisher. Zeros of the partition function for the Heisenberg, Ferroelectric, and general Ising models. $\mathcal{F}$. Math. Phys., 12(2):235-246, 1971.

[54] D. Weitz. Counting independent sets up to the tree threshold. In Proc. 38th ACM Symp. Theory Comput. (STOC), pages 140-149, 2006.

[55] C. N. Yang and T. D. Lee. Statistical theory of equations of state and phase transitions. I. Theory of condensation. Phys. Rev., 87(3):404-409, 1952.

[56] J. Zhang, H. Liang, and F. Bai. Approximating partition functions of the two-state spin system. Inf. Process. Lett., 111(14):702710, 2011 